1 DETERMINATION OF IN VITRO AND IN SILICO INDEXES FOR THE MODELLING OF

2 BLOOD-BRAIN BARRIER PARTITIONING OF DRUGS VIA MICELLAR AND

3 IMMOBILIZED ARTIFICIAL MEMBRANE LIQUID CHROMATOGRAPHY.

4

5 Giacomo Russo $^{1,2}$, Lucia Grumetto ${ }^{2}$, Roman Szucs $^{3}$, Francesco Barbato ${ }^{2}$, Frederic Lynen ${ }^{1 *}$.

$6 \quad *$ corresponding author

7 1. Separation Science Group, Department of Organic and Macromolecular Chemistry, Ghent

8 University, Krijgslaan 281, S4-bis, B-9000 Gent, Belgium

9 2. Dipartimento di Farmacia, Università degli Studi di Napoli Federico II, Via D. Montesano, 49 I1080131 Naples, Italy

11 3. Pfizer Global R\&D, Sandwich CT13 9NJ, Kent, United Kingdom 
BLOOD-BRAIN BARRIER PARTITIONING OF DRUGS VIA MICELLAR AND

IMMOBILIZED ARTIFICIAL MEMBRANE LIQUID CHROMATOGRAPHY.

ABSTRACT

18

In the present work, 79 structurally unrelated analytes were taken into account and their chromatographic retention coefficients, measured by Immobilized Artificial Membrane Liquid

Chromatography (IAM-LC), and by Micellar Liquid Chromatography (MLC) employing sodium dodecyl sulfate (SDS) as surfactant, were determined. Such indexes, along with topological and physico-chemical parameters calculated in silico, were subsequently used for the development of

Blood-Brain Barrier passage-predictive statistical models using partial least square (PLS) regression..

Highly significant relationships were observed either using IAM $\left(r^{2}(n-1)=0.78\right)$ or $\operatorname{MLC}\left(r^{2}(n-1)=\right.$

0.83) derived indexes along with in silico descriptors. This hybrid approach proved fast and effective in the development of highly predictive BBB passage oriented models and, therefore, it can be of interest for pharmaceutical industries as a high-throughput $\mathrm{BBB}$ penetration oriented screening method. Finally, it shed new light into the molecular mechanism involved in the BBB uptake of therapeutics.

31 Keywords: Immobilized Artificial Membrane; Micellar Liquid Chromatography; Blood-Brain Barrier 32 passage; Quantitative structure-activity relationships. 


\subsection{INTRODUCTION}

Pharmaceutical drug development is still a highly inefficient process: over one third of the failures

in drug candidate development is estimated to occur due to unsatisfactory pharmacokinetic

37 properties ${ }^{1}$, mainly regarding absorption, metabolism and toxicity and the attrition rates for Central

38 Nervous System (CNS) active drugs are even higher ${ }^{2}$. In fact, before reaching the blood circulation,

39 a drug diffuses through the biological barriers separating the circulating blood from the interstitial

40 fluid that surrounds the tissues. For orally administered drugs, this barrier is the intestinal

41 epithelium whereas the passage of drugs designed to act at the CNS level is further regulated by the

42 Blood-Brain Barrier (BBB). The $\mathrm{BBB}$ is one of the most complex and extensively studied

43 biological barriers, and its function is to preserve mammalian brain integrity against possible

44 injurious substances. It is made of endothelial cells, narrowly adherent one to the other to form tight

45 junctions, restricting the passage of solutes ${ }^{3,4}$. Indeed, drug transport is strongly limited by this peculiar biological structure to pure passive transcellular diffusion. In fact, the paracellular route,

47 i.e. the passage of actives through the gaps between each endothelial cell, is completely hindered.

48 Apart from active transport mechanisms, whose occurrence is difficult to predict on a solely

49 chemical structure basis, drugs can therefore cross the BBB only by the passive transcellular route.

50 Plenty of in vivo, ex vivo, and in vitro methods are available for measuring BBB partitioning of

51 analytes. Historically, one of the most used and reputed method is the determination of $\log$ BB 52 values ${ }^{5}$. Log BB is defined as:

53

$$
\log \mathrm{BB}=\log \frac{C_{\text {Brain }}}{C_{\text {Blood }}}
$$

in which $\mathrm{C}_{\mathrm{Brain}}$ is the concentration that the analyte realizes in the brain tissues, and $\mathrm{C}_{\mathrm{Blood}}$ is the

57 concentration that it realizes in the blood. However, this method involves the use of animal models, 
usually rodents, and does not provide any mechanistic information about the nature of the passage; furthermore, the method is time-consuming and potential source of ethical issues.

Methods based on the employment of cultured cell lines can also be effective; however, astrocytes cell cultures are often difficult to grow and recreating an in vitro environment similar to the in vivo BBB can be challenging even for the most experienced scientists. Caco-2 model based methods may also be an alternative; however, apart from the structural dissimilarities with the other cell cultures $^{6}$, they are difficult to standardize, complicating comparisons of data determined in different laboratories.

In silico methods, generally based on the calculation of physico-chemical parameters, yield various advantages. They are much faster to perform, allowing the screening of large libraries of compounds (even solutes not yet synthesized); in addition, they can assist in the elucidation of the molecular mechanisms involved in membrane permeation. However, they also suffer from several limitations including the aspect that they are unable to take into account all phenomena actually occurring in vivo ${ }^{7}$.

In vitro methods based on the use of biomimetic stationary phases coupled with high performance liquid chromatography (HPLC) have been used to surrogate BBB permeation data ${ }^{8,9}$. The main advantages are that they are much more reproducible and easier to perform and, albeit conceptually simple, they can be incidentally able to provide an in-depth understanding of the mechanisms involved in membrane barrier passage. Such biomimetic stationary phases include, for instance, Immobilized Artificial Membranes (IAM). IAM stationary phases are based on analogues of phosphatidylcholine, which is the major component of biological membranes, and chromatographic retention coefficients of the analytes on such stationary phases are assumed as direct measures of their phospholipophilicity ${ }^{10}$, i.e. their affinity for phospholipids. Such measures have been proven to be able to mirror various phenomena underlying membrane barrier passage ${ }^{8,11-13}$. In addition, other chromatographic indexes, whose drug BBB-penetration predictivity has been demonstrated ${ }^{14-16}$, include those achieved by Micellar Liquid Chromatography (MLC) technique. This technique is 
based on the addition of surfactants to an aqueous mobile phase at concentrations higher than their critical micelle concentrations $(\mathrm{CMC})^{17}$ resulting in the formation of micelles acting as a partition phase. Both IAM and MLC chromatographic indexes, mainly if combined with in silico calculated descriptors, have demonstrated effectiveness in the prediction of BBB drug penetration ${ }^{16}$. However, the methods proposed are still too time-consuming to meet the demands of pharmaceutical companies and their suitability should be confirmed on larger set of analytes.

The aim of the present work has been the development of drug BBB penetration oriented statistical models based on analytical indexes, achieved on biomimetic conditions by medium/highthroughput methods, along with in silico calculated descriptors. To the best of our knowledge, this is the study based on the highest number of compounds among those employing IAM and MLC data to predict drug pharmacokinetic properties.

Therefore, particular attention is set on:

i) the setup of medium/high-throughput methods for the achievement of both IAM and MLC indexes;

ii) the validation of such parameters by developing statistical models for the prediction of BBB penetration $(\log \mathrm{BB})$ by using the chromatographic indexes along with in silico calculated descriptors;

iii) the elucidation of the molecular mechanism involved in BBB passive diffusion of drugs;

$i v)$ the possibility of taking into account, by molecular docking studies, the occurrence of active efflux mechanisms.

In the present work, 79 structurally unrelated analytes have been taken into account and their chromatographic retention coefficients, measured by high-throughput IAM-LC and MLC methods, the latter employing sodium dodecyl sulfate (SDS) as surfactant, were determined. Such indexes have subsequently been used for the development of BBB-passage predictive statistical models using partial least squares (PLS) automatic regression along with physico-chemical parameters, calculated in silico. Such hybrid approach was aimed at combining the speediness in the 
110 achievement of computational chemistry derived topological and physico-chemical parameters with

111 the improved predictivity of the in vitro methods.

\subsection{RESULTS AND DISCUSSION}

114 The IAM-LC and MLC chromatographic retention coefficients, as well as the $\mathrm{pKa}, \log \mathrm{BB}$ values, 115 UV wavelengths of the experimental determinations and suppliers, are presented in Table 1. In 116 MLC, the highest retained compound (triprolidine) eluted within 33.0 minutes, whereas in IAM-LC 117 the maximum run time was 37.0 minutes (fluphenazine). However, by performing either the MLC 118 or the IAM-LC analytical methods, $85 \%$ of the compounds of the dataset eluted within 15.0 minutes 119 and a preliminary estimate, as an order of magnitude, of the retention times expected can be easily 120 performed based on the calculation of $\log \mathrm{D}^{7.4}$ values of each compound present in the dataset. Two 121 chromatographic runs for each technique are reported in Figure 1 (MLC) and Figure 2 (IAM-LC). 122 The $\log \mathrm{BB}$ values span a very large range (from -2.00 to +1.51 ) as the analytes to be included in 123 the dataset were selected to include both CNS inactive (e.g. norfloxacin, nitrofurantoin) and CNS 124 active (e.g. fluphenazine, desipramine) drugs. The P-gp affinities, expressed in $\mathrm{kcal} \cdot \mathrm{mol}^{-1}$, of the 125 drugs considered are listed in Table 2A and Table 2B. They were incorporated in each of the 126 following steps to model even the BBB passage of analytes undergoing P-gp effux mechanisms. 
129 Figure 1. MLC chromatograms achieved for the analytes A) Imipramine (50 $\mu \mathrm{g} \mathrm{mL}^{-1}$ in methanol) 130 and B) Ethylbenzene (50 $\mu \mathrm{g} \mathrm{mL}^{-1}$ in methanol) employing a mobile phase consisting of an aqueous 131 solution of $0.05 \mathrm{~mol} \cdot \mathrm{L}^{-1}$ sodium dodecyl sulfate. The detailed experimental conditions are reported in paragraph 4.0 .

133

134

135

136

137

138
30.0

B

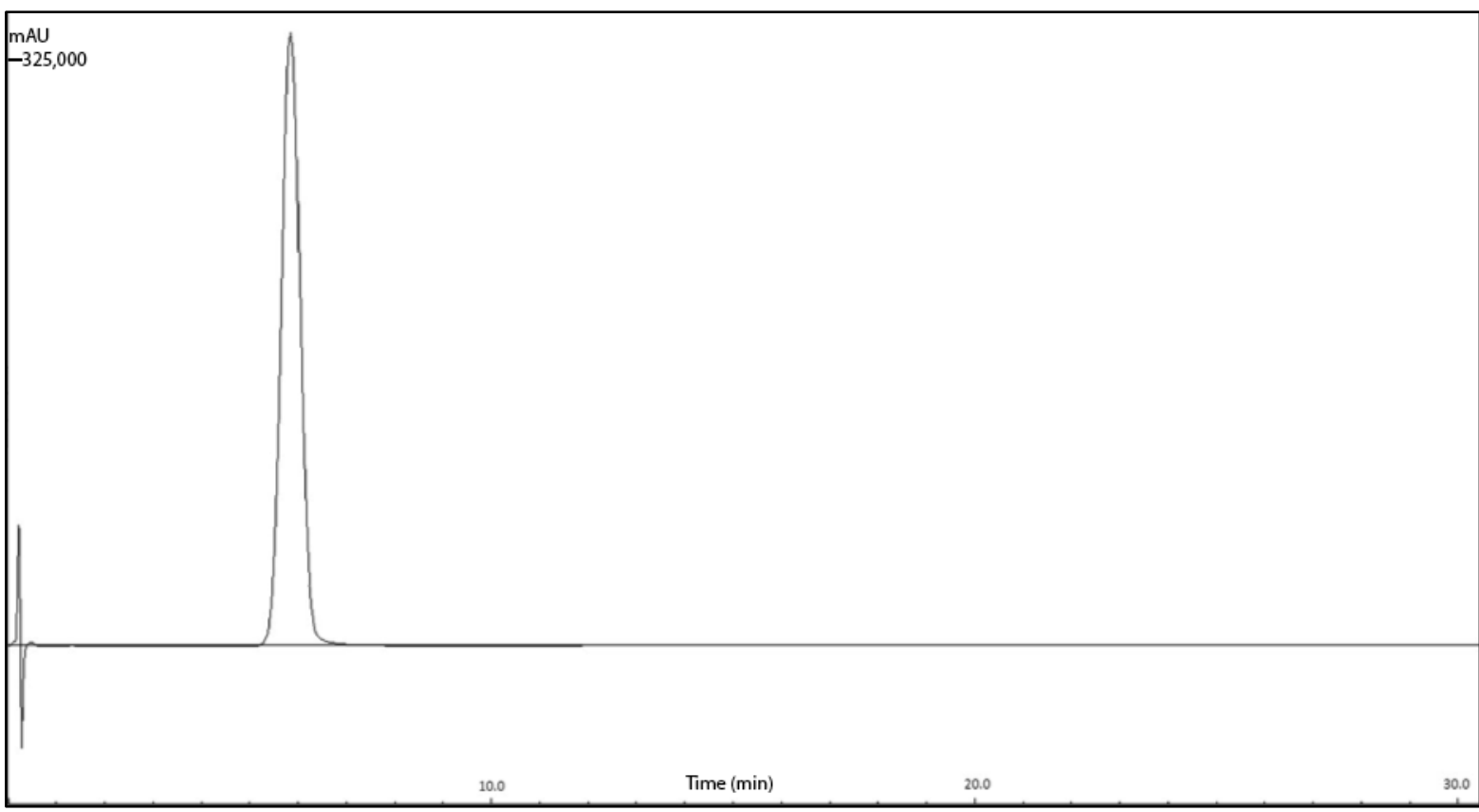


142 Figure 2. IAM chromatograms achieved for the analytes A) Paroxetine (50 $\mu \mathrm{g} \mathrm{mL} \mathrm{m}^{-1}$ in methanol) and B) Diclofenac $\left(50 \mu \mathrm{g} \mathrm{mL}{ }^{-1}\right.$ in methanol). The mobile phase was a solution $70 / 30 \mathrm{v} / \mathrm{v}$

144 Dulbecco's phosphate-buffered saline (DPBS) / methanol. The detailed experimental conditions are 145 reported in paragraph 4.0.

146

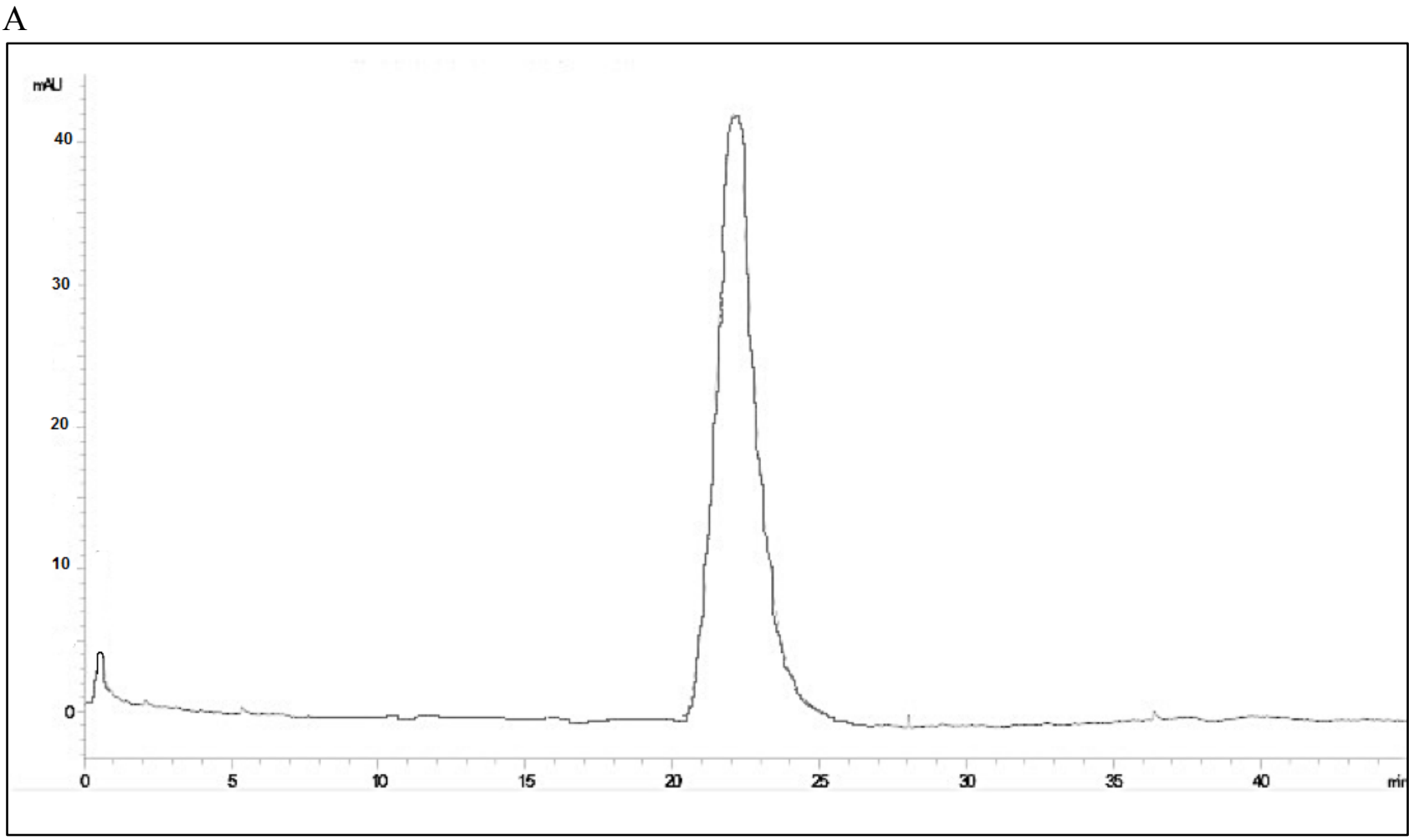

B

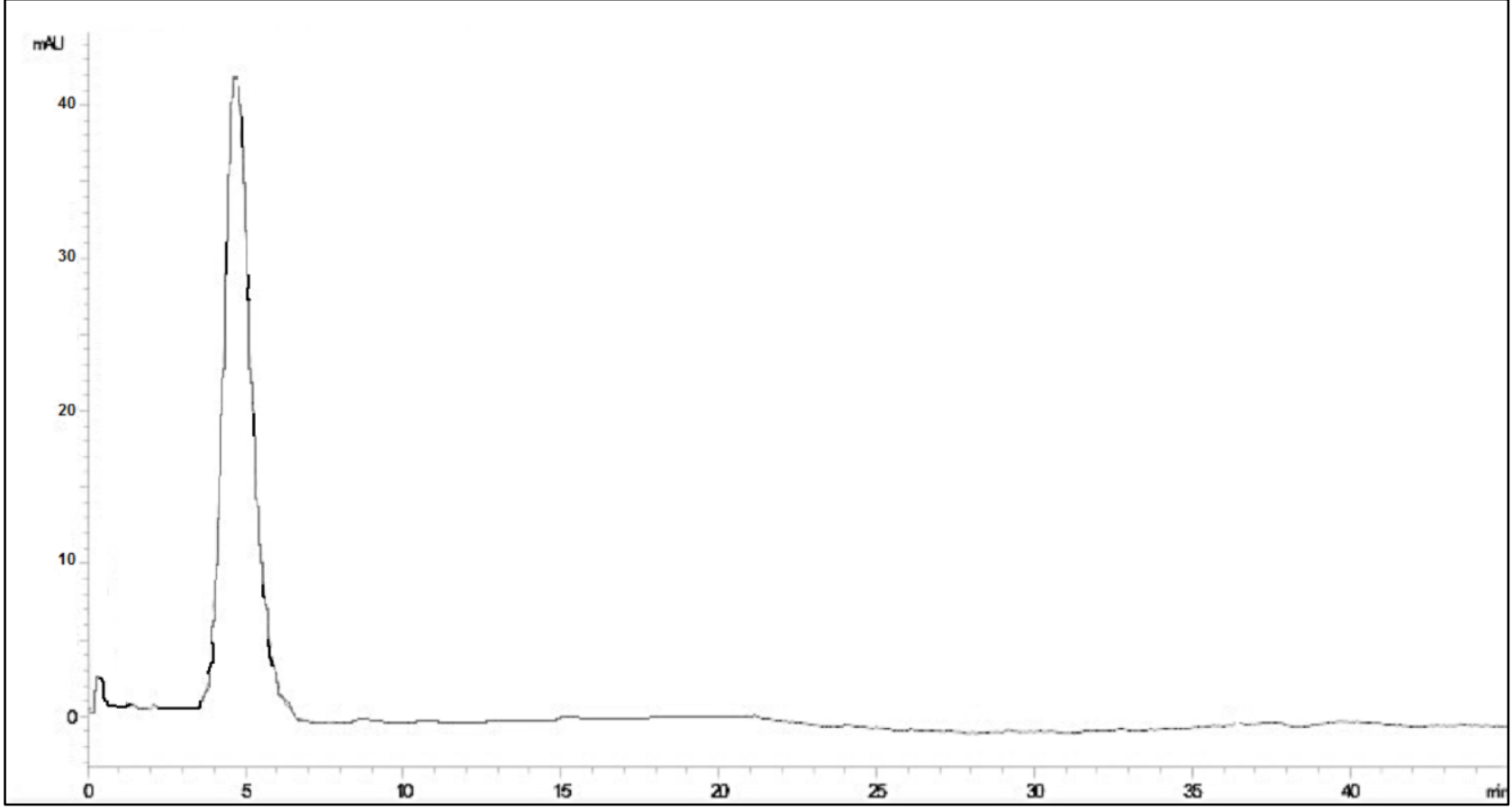


Table 1. $\mathrm{pKa}$ values, $\log \mathrm{k}_{\mathrm{w}}{ }^{\mathrm{SDS}}, \log \mathrm{k}_{30 \% \mathrm{MeOH}}{ }^{\mathrm{IAM}}$ indexes, $\log \mathrm{BB}$ values, experimental UV wavelengths and suppliers for the analytes taken into account.

153

\begin{tabular}{|c|c|c|c|c|c|c|}
\hline Analyte & $\mathrm{pKa}$ & $\underset{\mathrm{SDS}}{\log k_{\mathrm{w}}}$ & 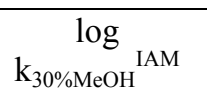 & $\log \mathrm{BB}$ & $\begin{array}{l}\text { UV wavelength } \\
(\mathrm{nm})\end{array}$ & Supplier \\
\hline $\begin{array}{l}2- \\
\text { (Methylamino)pyridine }\end{array}$ & - & 1.611 & -0.164 & $-0.30^{18}$ & 254 & $\begin{array}{l}\text { Sigma- } \\
\text { Aldrich }\end{array}$ \\
\hline $\begin{array}{l}\text { 2,2,2-trifluoroethyl } \\
\text { vinyl ether }\end{array}$ & - & 0.929 & -0.142 & $0.13^{18}$ & 210 & $\begin{array}{l}\text { Sigma- } \\
\text { Aldrich }\end{array}$ \\
\hline 2,6-diisopropylphenol & - & 1.688 & 1.097 & $0.91^{19}$ & 210 & $\begin{array}{r}\text { TCI } \\
\text { Europe }\end{array}$ \\
\hline Acetaminophen & 9.69 & -0.092 & -0.204 & $-1.00^{19}$ & 254 & $\begin{array}{r}\text { Acros } \\
\text { Organics }\end{array}$ \\
\hline Acetylsalicylic acid & 3.50 & -0.301 & -0.274 & $-1.30^{19}$ & 230 & $\begin{array}{r}\text { Acros } \\
\text { Organics }\end{array}$ \\
\hline Aminopyrine & 5.03 & 1.486 & -0.206 & $0.00^{19}$ & 254 & $\begin{array}{l}\text { Sigma- } \\
\text { Aldrich }\end{array}$ \\
\hline Amitriptyline & 9.17 & 2.230 & 1.606 & $1.30^{19}$ & 254 & $\begin{array}{r}\text { TCI } \\
\text { Europe }\end{array}$ \\
\hline Amobarbital & $7.48 / 11.15^{*}$ & 1.208 & 0.059 & $0.04^{19}$ & 210 & $\begin{array}{l}\text { Sigma- } \\
\text { Aldrich }\end{array}$ \\
\hline Antipyrine & 1.44 & 1.059 & -0.277 & $-0.10^{19}$ & 240 & $\begin{array}{r}\text { Acros } \\
\text { Organics }\end{array}$ \\
\hline Atenolol & 9.19 & 1.156 & -0.162 & $-1.00^{19}$ & 270 & $\begin{array}{r}\text { Acros } \\
\text { Organics }\end{array}$ \\
\hline Benzene & - & 1.202 & 0.036 & $0.37^{20}$ & 210 & $\begin{array}{r}\text { TCI } \\
\text { Europe }\end{array}$ \\
\hline Betahistine & 7.84 & 0.125 & -0.193 & $-0.30^{21}$ & 254 & $\begin{array}{r}\text { Acros } \\
\text { Organics }\end{array}$ \\
\hline Caffeine & 0.60 & 0.910 & -0.284 & $-0.06^{19}$ & 210 & $\begin{array}{r}\text { Acros } \\
\text { Organics }\end{array}$ \\
\hline Carbamazepine & - & 1.191 & 0.210 & $0.00^{19}$ & 210 & $\begin{array}{r}\text { Acros } \\
\text { Organics }\end{array}$ \\
\hline Celecoxib & 9.38 & 1.461 & 1.613 & $-1.00^{21}$ & 254 & $\begin{array}{r}\text { Acros } \\
\text { Organics }\end{array}$ \\
\hline Chlorambucil & 4.60 & 0.787 & 0.308 & $-1.70^{19}$ & 254 & $\begin{array}{r}\text { TCI } \\
\text { Europe }\end{array}$ \\
\hline Chlorpromazine & 9.50 & 2.169 & 2.038 & $1.36^{21}$ & 254 & $\begin{array}{r}\text { TCI } \\
\text { Europe }\end{array}$ \\
\hline Cimetidine & 7.01 & 1.003 & -0.177 & $-1.42^{19}$ & 210 & $\begin{array}{r}\text { TCI } \\
\text { Europe }\end{array}$ \\
\hline Citalopram & 9.22 & 1.832 & 1.005 & $0.48^{21}$ & 210 & $\begin{array}{r}\text { TCI } \\
\text { Europe }\end{array}$ \\
\hline Clonidine & 8.08 & 1.436 & 0.171 & $0.11^{19}$ & 270 & $\begin{array}{r}\text { TCI } \\
\text { Europe }\end{array}$ \\
\hline Clozapine & 7.90 & 1.784 & 1.529 & $0.60^{21}$ & 254 & $\begin{array}{r}\text { TCI } \\
\text { Europe }\end{array}$ \\
\hline Cotinine & - & 1.424 & -0.260 & $-0.32^{19}$ & 260 & $\begin{array}{r}\text { TCI } \\
\text { Europe }\end{array}$ \\
\hline Cyclobenzaprine & 8.47 & 2.092 & 1.607 & $1.08^{21}$ & 230 & $\begin{array}{r}\text { TCI } \\
\text { Europe }\end{array}$ \\
\hline Desipramine & 10.28 & 2.144 & 1.536 & $1.20^{19}$ & 254 & $\begin{array}{l}\text { Sigma- } \\
\text { Aldrich }\end{array}$ \\
\hline Diclofenac & 3.99 & 0.602 & 0.024 & $-1.70^{21}$ & 254 & $\begin{array}{r}\text { Acros } \\
\text { Organics }\end{array}$ \\
\hline Diphenhydramine & 8.86 & 2.077 & 0.858 & $1.20^{21}$ & 210 & $\begin{array}{r}\text { Acros } \\
\text { Organics }\end{array}$ \\
\hline Domperidone & 9.68 & 1.937 & 1.562 & $-0.78^{19}$ & 270 & $\begin{array}{r}\mathrm{TCI} \\
\text { Europe } \\
\end{array}$ \\
\hline
\end{tabular}




\begin{tabular}{|c|c|c|c|c|c|c|}
\hline Donepezil & $8.54^{*}$ & 1.968 & 0.858 & $0.89^{22}$ & 210 & $\begin{array}{r}\text { Acros } \\
\text { Organics }\end{array}$ \\
\hline Eserine & 8.17 & 1.656 & 0.030 & $0.08^{19}$ & 240 & $\begin{array}{r}\text { TCI } \\
\text { Europe }\end{array}$ \\
\hline Ethosuximide & 9.27 & 0.545 & -0.228 & $0.04^{21}$ & 210 & $\begin{array}{r}\text { Acros } \\
\text { Organics }\end{array}$ \\
\hline Ethylbenzene & - & 1.588 & 0.600 & $0.26^{20}$ & 210 & $\begin{array}{r}\text { Acros } \\
\text { Organics }\end{array}$ \\
\hline Fluphenazine & $7.84 / 2.08^{*}$ & 2.207 & 2.066 & $1.51^{19}$ & 254 & $\begin{array}{l}\text { Sigma- } \\
\text { Aldrich }\end{array}$ \\
\hline Haloperidol & 8.29 & 2.366 & 1.483 & $1.34^{22}$ & 254 & $\begin{array}{r}\text { TCI } \\
\text { Europe }\end{array}$ \\
\hline Halothane & - & 1.215 & 0.152 & $0.35^{20}$ & 210 & $\begin{array}{l}\text { Sigma- } \\
\text { Aldrich }\end{array}$ \\
\hline Hexobarbital & 8.20 & 1.284 & -0.008 & $0.10^{19}$ & 254 & $\begin{array}{l}\text { Sigma- } \\
\text { Aldrich }\end{array}$ \\
\hline Hydroxyzine & $7.52 / 1.58^{*}$ & 2.038 & 1.337 & $0.90^{21}$ & 210 & $\begin{array}{l}\text { Sigma- } \\
\text { Aldrich }\end{array}$ \\
\hline Ibuprofen & 4.24 & 0.626 & 0.090 & $-0.18^{19}$ & 270 & $\begin{array}{r}\text { Acros } \\
\text { Organics }\end{array}$ \\
\hline Imipramine & 9.52 & 2.190 & 1.452 & $1.30^{19}$ & 240 & $\begin{array}{r}\text { Acros } \\
\text { Organics }\end{array}$ \\
\hline Indomethacin & 4.13 & 0.647 & -0.257 & $-1.26^{19}$ & 210 & $\begin{array}{r}\text { TCI } \\
\text { Europe }\end{array}$ \\
\hline Ketorolac & 3.84 & -0.097 & -0.500 & $-2.00^{21}$ & 300 & $\begin{array}{r}\text { TCI } \\
\text { Europe }\end{array}$ \\
\hline Lamotrigine & 5.36 & 1.316 & -0.006 & $0.48^{23}$ & 220 & $\begin{array}{r}\text { Acros } \\
\text { Organics }\end{array}$ \\
\hline Levofloxacin & $6.20 / 5.45^{*}$ & 1.388 & -0.099 & $-0.70^{21}$ & 290 & $\begin{array}{r}\text { TCI } \\
\text { Europe }\end{array}$ \\
\hline Metanol & - & 0.000 & -0.447 & $0.02^{23}$ & 210 & $\begin{array}{l}\text { Sigma- } \\
\text { Aldrich }\end{array}$ \\
\hline Metoclopramide & 9.71 & 1.610 & 0.346 & $0.08^{21}$ & 210 & $\begin{array}{r}\text { TCI } \\
\text { Europe }\end{array}$ \\
\hline Metoprolol & 9.56 & 1.771 & 0.198 & $1.15^{22}$ & 220 & $\begin{array}{r}\text { TCI } \\
\text { Europe }\end{array}$ \\
\hline Mianserin & 6.92 & 2.152 & 1.456 & $0.99^{19}$ & 280 & $\begin{array}{r}\text { TCI } \\
\text { Europe }\end{array}$ \\
\hline Naproxen & 4.14 & 0.153 & -0.090 & $-1.70^{21}$ & 254 & $\begin{array}{r}\text { Acros } \\
\text { Organics }\end{array}$ \\
\hline Nicotine & 8.11 & 1.969 & -0.139 & $0.40^{20}$ & 210 & $\begin{array}{r}\text { Acros } \\
\text { Organics }\end{array}$ \\
\hline Nitrofurantoin & 7.05 & -0.074 & -0.447 & $-2.00^{21}$ & 254 & $\begin{array}{r}\text { Acros } \\
\text { Organics }\end{array}$ \\
\hline Norfloxacin & $8.68 / 5.77^{*}$ & 1.332 & -0.062 & $-1.00^{21}$ & 280 & $\begin{array}{r}\text { Acros } \\
\text { Organics }\end{array}$ \\
\hline Nortriptyline & 10.13 & 2.169 & 1.639 & $1.04^{21}$ & 254 & $\begin{array}{r}\text { TCI } \\
\text { Europe }\end{array}$ \\
\hline Olanzapine & 7.80 & 1.825 & 0.843 & $0.80^{21}$ & 254 & $\begin{array}{r}\text { TCI } \\
\text { Europe }\end{array}$ \\
\hline Omeprazole & $9.29 / 4.77 *$ & 1.591 & -0.229 & $-0.82^{19}$ & 300 & $\begin{array}{r}\text { TCI } \\
\text { Europe }\end{array}$ \\
\hline Oxazepam & - & 1.420 & 0.707 & $0.61^{19}$ & 230 & $\begin{array}{l}\text { Sigma- } \\
\text { Aldrich }\end{array}$ \\
\hline Paroxetine & 9.77 & 2.104 & 1.796 & $0.48^{21}$ & 210 & $\begin{array}{r}\text { TCI } \\
\text { Europe }\end{array}$ \\
\hline Pentobarbital & 8.18 & 1.243 & 0.103 & $0.12^{19}$ & 210 & $\begin{array}{l}\text { Sigma- } \\
\text { Aldrich }\end{array}$ \\
\hline Phenylbutazone & 4.34 & 0.996 & 0.273 & $-0.52^{19}$ & 240 & $\begin{array}{r}\text { Acros } \\
\text { Organics }\end{array}$ \\
\hline Phenytoin & 8.28 & 1.311 & 0.382 & $-0.04^{19}$ & 210 & $\begin{array}{r}\text { Acros } \\
\text { Organics }\end{array}$ \\
\hline
\end{tabular}




\begin{tabular}{|c|c|c|c|c|c|c|}
\hline Pindolol & 9.54 & 0.811 & 0.312 & $0.30^{21}$ & 210 & $\begin{array}{l}\text { Sigma- } \\
\text { Aldrich }\end{array}$ \\
\hline Primidone & - & 0.710 & -0.152 & $-0.07^{21}$ & 210 & $\begin{array}{r}\text { TCI } \\
\text { Europe }\end{array}$ \\
\hline Promazine & 9.36 & 2.030 & 1.643 & $1.23^{20}$ & 254 & $\begin{array}{l}\text { Sigma- } \\
\text { Aldrich }\end{array}$ \\
\hline Promethazine & 9.00 & 2.040 & 1.613 & $1.30^{24}$ & 254 & $\begin{array}{r}\text { TCI } \\
\text { Europe }\end{array}$ \\
\hline Propranolol & 9.16 & 2.028 & 0.992 & $0.85^{21}$ & 290 & $\begin{array}{r}\text { Acros } \\
\text { Organics }\end{array}$ \\
\hline Quinidine & 8.56 & 2.245 & 0.982 & $0.33^{22}$ & 230 & $\begin{array}{r}\text { Acros } \\
\text { Organics }\end{array}$ \\
\hline Ranitidine & 8.33 & 1.233 & -0.239 & $-1.23^{19}$ & 230 & $\begin{array}{l}\text { TCI } \\
\text { Europe }\end{array}$ \\
\hline Rifampicin & 1.70 & 1.900 & 0.990 & $-1.52^{21}$ & 230 & $\begin{array}{r}\text { TCI } \\
\text { Europe }\end{array}$ \\
\hline Ropinirole & 10.17 & 1.685 & 0.326 & $0.25^{19}$ & 254 & $\begin{array}{l}\text { Sigma- } \\
\text { Aldrich }\end{array}$ \\
\hline Salicylic acid & 2.82 & -0.280 & -0.302 & $-1.10^{19}$ & 300 & $\begin{array}{r}\text { Acros } \\
\text { Organics }\end{array}$ \\
\hline Theobromine & - & 0.347 & -0.284 & $-0.28^{19}$ & 270 & $\begin{array}{r}\text { Acros } \\
\text { Organics }\end{array}$ \\
\hline Theophylline & - & 0.447 & -0.218 & $-0.29^{19}$ & 270 & $\begin{array}{r}\text { Acros } \\
\text { Organics }\end{array}$ \\
\hline Toluene & - & 1.459 & 0.330 & $0.37^{20}$ & 210 & $\begin{array}{r}\text { Acros } \\
\text { Organics }\end{array}$ \\
\hline Tramadol & 9.41 & 1.692 & 0.256 & $0.70^{21}$ & 210 & $\begin{array}{l}\text { Sigma- } \\
\text { Aldrich }\end{array}$ \\
\hline Trazodone & 7.30 & 2.223 & 0.780 & $0.30^{21}$ & 210 & $\begin{array}{l}\text { Sigma- } \\
\text { Aldrich }\end{array}$ \\
\hline Triprolidine & 8.64 & 2.493 & 0.789 & $0.78^{21}$ & 230 & $\begin{array}{l}\text { Sigma- } \\
\text { Aldrich }\end{array}$ \\
\hline Valproic acid & 4.54 & 0.001 & -0.279 & $-0.84^{19}$ & 210 & $\begin{array}{r}\text { Acros } \\
\text { Organics }\end{array}$ \\
\hline Venlafaxine & 9.67 & 1.900 & 0.429 & $0.48^{25}$ & 230 & $\begin{array}{r}\text { Acros } \\
\text { Organics }\end{array}$ \\
\hline Verapamil & 8.68 & 2.271 & 1.169 & $-0.52^{19}$ & 210 & $\begin{array}{r}\text { Acros } \\
\text { Organics }\end{array}$ \\
\hline Zidovudine & 9.40 & 0.271 & -0.264 & $-1.00^{20}$ & 270 & $\begin{array}{r}\text { Acros } \\
\text { Organics }\end{array}$ \\
\hline Zolmitriptan & 9.55 & 0.974 & -0.159 & $-1.40^{21}$ & 220 & $\begin{array}{r}\text { TCI } \\
\text { Europe }\end{array}$ \\
\hline
\end{tabular}


158 Table 2A. Minimum and most populated values, expressed in $\mathrm{kcal} \mathrm{mol}^{-1}$, of the cluster affinities of the analytes for the first four (from 1 to 4 ) discrete binding sites located on the P-gp.

\begin{tabular}{|c|c|c|c|c|c|c|c|c|}
\hline Analyte & $\begin{array}{l}\text { P-gp } \\
1 \text { Min }\end{array}$ & $\begin{array}{l}\text { P-gp } \\
1 \text { MP }\end{array}$ & $\begin{array}{l}\text { P-gp } \\
2 \text { Min }\end{array}$ & $\begin{array}{l}\text { P-gp } \\
2 \text { MP }\end{array}$ & $\begin{array}{l}\text { P-gp } \\
3 \text { Min }\end{array}$ & $\begin{array}{l}\text { P-gp } \\
3 \mathrm{MP}\end{array}$ & $\begin{array}{l}\text { P-gp } \\
4 \text { Min }\end{array}$ & $\begin{array}{l}\text { P-gp } \\
4 \text { MP }\end{array}$ \\
\hline 2-(Methylamino)pyridine & -3.03 & -3.03 & -3.61 & -3.61 & -3.62 & -3.62 & -3.63 & -3.63 \\
\hline 2,2,2-trifluoroethyl vinyl ether & -1.72 & -1.72 & -2.09 & -2.09 & -1.85 & -1.85 & -1.98 & -1.94 \\
\hline 2,6-diisopropylphenol & -4.42 & -4.42 & -5.36 & -5.36 & -5.55 & -5.55 & -5.36 & -5.36 \\
\hline Acetaminophen & -3.30 & -3.30 & -4.72 & -4.72 & -4.13 & -4.08 & -4.85 & -4.85 \\
\hline Acetylsalicylic acid & -3.57 & -3.57 & -4.47 & -4.36 & -4.48 & -4.48 & -4.22 & -3.95 \\
\hline Aminopyrine & -4.43 & -4.30 & -5.66 & -5.66 & -5.63 & -5.63 & -5.70 & -5.70 \\
\hline Amitriptyline & -6.09 & -5.02 & -7.29 & -7.15 & -6.39 & -6.39 & -7.22 & -7.22 \\
\hline Amobarbital & -4.14 & -4.06 & -5.30 & -5.00 & -4.83 & -4.83 & -5.05 & -5.05 \\
\hline Antipyrine & -4.33 & -4.33 & -5.61 & -5.61 & -5.31 & -5.31 & -5.61 & -5.44 \\
\hline Atenolol & -3.81 & -3.34 & -5.74 & -3.99 & -3.82 & -3.40 & -4.83 & -4.70 \\
\hline Benzene & -2.72 & -2.72 & -3.31 & -3.31 & -3.31 & -3.31 & -3.31 & -3.31 \\
\hline Betahistine & -3.06 & -3.06 & -3.68 & -3.68 & -3.32 & -3.14 & -3.70 & -3.70 \\
\hline Caffeine & -3.80 & -3.80 & -4.60 & -4.60 & -4.23 & -4.23 & -4.33 & -4.33 \\
\hline Carbamazepine & -5.84 & -5.82 & -7.09 & -7.09 & -6.12 & -6.12 & -7.07 & -7.07 \\
\hline Celecoxib & -4.30 & -4.30 & -7.01 & -6.98 & -4.73 & -4.12 & -7.18 & -7.18 \\
\hline Chlorambucil & -3.77 & -3.77 & -5.13 & -4.81 & -5.19 & -5.19 & -5.16 & -5.16 \\
\hline Chlorpromazine & -5.38 & -4.84 & -7.24 & -7.24 & -6.57 & -6.57 & -7.26 & -7.26 \\
\hline Cimetidine & -3.27 & -2.95 & -4.74 & -4.60 & -4.10 & -4.02 & -4.70 & -4.64 \\
\hline Citalopram & -4.75 & -4.45 & -6.41 & -6.14 & -4.93 & -4.93 & -7.16 & -6.86 \\
\hline Clonidine & -4.34 & -4.34 & -5.41 & -5.41 & -5.44 & -5.44 & -5.40 & -5.40 \\
\hline Clozapine & -5.10 & -5.05 & -7.01 & -7.01 & -5.36 & -5.36 & -7.00 & -6.98 \\
\hline Cotinine & -3.93 & -3.87 & -5.14 & -4.79 & -4.92 & -4.92 & -4.82 & -4.82 \\
\hline Cyclobenzaprine & -6.32 & -5.14 & -7.18 & -7.04 & -6.94 & -6.94 & -7.14 & -7.14 \\
\hline Desipramine & -5.75 & -5.43 & -6.80 & -6.62 & -6.26 & -6.08 & -6.53 & -6.53 \\
\hline Diclofenac & -5.23 & -4.96 & -6.34 & -6.13 & -6.49 & -6.19 & -6.14 & -6.05 \\
\hline Diphenhydramine & -4.35 & -3.91 & -5.63 & -5.63 & -5.14 & -5.14 & -5.61 & -5.61 \\
\hline Domperidone & -5.23 & -4.82 & -7.12 & -6.98 & -6.39 & -6.39 & -7.18 & -7.18 \\
\hline Donepezil & -6.05 & -6.05 & -7.70 & -7.46 & -6.69 & -6.67 & -7.72 & -7.65 \\
\hline Eserine & -4.88 & -4.88 & -6.01 & -5.87 & -5.45 & -5.45 & -5.91 & -5.91 \\
\hline Ethosuximide & -3.62 & -3.62 & -4.22 & -4.22 & -4.47 & -4.39 & -4.42 & -4.42 \\
\hline Ethylbenzene & -3.34 & -3.34 & -4.22 & -4.22 & -4.07 & -4.07 & -4.07 & -4.07 \\
\hline Fluphenazine & -4.81 & -3.58 & -6.75 & -6.75 & -4.30 & -4.12 & -6.60 & -5.82 \\
\hline Haloperidol & -4.35 & -4.17 & -6.25 & -6.25 & -5.60 & -5.60 & -7.23 & -6.20 \\
\hline Halothane & -2.12 & -2.12 & -2.76 & -2.75 & -2.74 & -2.72 & -2.66 & -2.64 \\
\hline Hexobarbital & -4.85 & -4.79 & -6.02 & -6.02 & -5.13 & -4.97 & -6.03 & -6.03 \\
\hline Hydroxyzine & -4.24 & -3.67 & -6.41 & -5.43 & -4.05 & -3.66 & -6.19 & -5.84 \\
\hline Ibuprofen & -4.37 & -4.37 & -5.52 & -5.43 & -5.88 & -5.88 & -5.43 & -5.34 \\
\hline Imipramine & -5.34 & -5.34 & -6.68 & -6.68 & -5.76 & -4.64 & -6.68 & -6.13 \\
\hline Indomethacin & -5.67 & -5.53 & -7.02 & -7.02 & -7.28 & -7.28 & -7.37 & -7.37 \\
\hline Ketorolac & -5.22 & -5.22 & -6.61 & -6.61 & -6.55 & -6.41 & -6.62 & -6.62 \\
\hline Lamotrigine & -4.49 & -3.92 & -5.84 & -5.84 & -4.88 & -4.56 & -5.36 & -5.36 \\
\hline Levofloxacin & -4.45 & -4.45 & -5.80 & -5.54 & -5.80 & -5.23 & -6.07 & -5.85 \\
\hline Metanol & -1.40 & -1.33 & -1.43 & -1.43 & -1.33 & -1.33 & -1.40 & -1.40 \\
\hline
\end{tabular}




\begin{tabular}{|c|c|c|c|c|c|c|c|c|}
\hline Metoclopramide & -3.47 & -3.47 & -5.19 & -3.92 & -3.71 & -3.07 & -4.52 & -4.13 \\
\hline Metoprolol & -3.58 & -3.35 & -4.63 & -4.06 & -3.54 & -2.87 & -4.39 & -4.18 \\
\hline Mianserin & -5.23 & -5.23 & -7.06 & -7.06 & -6.05 & -5.98 & -7.11 & -7.11 \\
\hline Naproxen & -4.82 & -4.82 & -5.99 & -5.99 & -6.03 & -5.91 & -6.03 & -6.03 \\
\hline Nicotine & -4.02 & -4.02 & -4.70 & -4.69 & -4.50 & -4.50 & -4.70 & -4.70 \\
\hline Nitrofurantoin & -4.10 & -4.10 & -5.33 & -5.33 & -5.18 & -5.18 & -5.32 & -5.32 \\
\hline Norfloxacin & -3.83 & -3.83 & -5.59 & -5.59 & -5.51 & -5.51 & -5.75 & -5.63 \\
\hline Nortriptyline & -6.35 & -6.35 & -7.07 & -6.86 & -6.44 & -6.44 & -7.00 & -7.00 \\
\hline Olanzapine & -4.60 & -4.60 & -6.71 & -6.62 & -5.47 & -5.29 & -6.68 & -6.68 \\
\hline Omeprazole & -5.26 & -5.20 & -6.96 & -6.76 & -6.65 & -6.41 & -7.16 & -6.92 \\
\hline Oxazepam & -5.29 & -5.29 & -6.90 & -6.90 & -6.16 & -6.16 & -6.89 & -6.89 \\
\hline Paroxetine & -4.94 & -4.94 & -6.95 & -6.84 & -6.42 & -5.67 & -6.83 & -6.29 \\
\hline Pentobarbital & -3.88 & -3.88 & -4.76 & -4.76 & -4.41 & -4.41 & -4.91 & -4.91 \\
\hline Phenylbutazone & -5.53 & -5.53 & -7.27 & -7.27 & -6.02 & -5.12 & -7.45 & -6.69 \\
\hline Phenytoin & -5.00 & -5.00 & -6.56 & -6.56 & -5.39 & -5.00 & -6.55 & -6.55 \\
\hline Pindolol & -4.17 & -4.13 & -5.43 & -4.86 & -4.56 & -4.26 & -5.47 & -4.60 \\
\hline Primidone & -4.55 & -4.55 & -5.19 & -5.19 & -4.88 & -4.71 & -5.50 & -5.50 \\
\hline Promazine & -5.58 & -5.58 & -6.79 & -6.50 & -6.32 & -6.32 & -6.50 & -5.84 \\
\hline Promethazine & -4.99 & -4.80 & -6.78 & -6.49 & -6.60 & -5.83 & -6.51 & -6.42 \\
\hline Propranolol & -4.60 & -4.38 & -6.33 & -5.54 & -4.79 & -4.22 & -6.10 & -5.39 \\
\hline Quinidine & -5.72 & -5.72 & -7.43 & -7.43 & -5.26 & -4.56 & -7.77 & -7.77 \\
\hline Ranitidine & -2.77 & -2.65 & -4.11 & -3.91 & -4.42 & -2.88 & -4.31 & -3.48 \\
\hline Rifampicin & -7.10 & -6.50 & -4.48 & -4.32 & -6.80 & -5.95 & -7.22 & -6.59 \\
\hline Ropinirole & -4.02 & -4.01 & -6.30 & -5.55 & -4.72 & -3.94 & -5.94 & -5.52 \\
\hline Salicylic acid & -3.09 & -3.09 & -3.69 & -3.69 & -4.00 & -4.00 & -3.74 & -3.71 \\
\hline Theobromine & -3.47 & -3.47 & -4.54 & -4.54 & -4.08 & -4.08 & -4.54 & -4.54 \\
\hline Theophylline & -3.63 & -3.63 & -4.43 & -4.43 & -3.88 & -3.87 & -4.43 & -4.43 \\
\hline Toluene & -3.08 & -3.08 & -3.81 & -3.81 & -3.77 & -3.77 & -3.74 & -3.74 \\
\hline Tramadol & -4.89 & -4.89 & -5.94 & -5.20 & -5.15 & -3.77 & -5.71 & -5.3 \\
\hline Trazodone & -5.37 & -5.00 & -7.30 & -5.74 & -5.79 & -6.47 & -7.09 & -6.06 \\
\hline Triprolidine & -5.03 & -4.92 & -7.19 & -7.11 & -5.36 & -5.36 & -6.96 & -6.88 \\
\hline Valproic acid & -2.78 & -2.78 & -3.56 & -3.56 & -3.81 & -3.81 & -3.43 & -3.4 \\
\hline Venlafaxine & -4.81 & -4.54 & -6.07 & -5.82 & -4.54 & -4.09 & -6.47 & -6.08 \\
\hline Verapamil & -3.93 & -3.54 & -6.56 & -6.37 & -5.28 & -4.48 & -6.58 & -6.1 \\
\hline Zidovudine & -3.45 & -3.21 & -5.14 & -5.14 & -3.56 & -3.27 & -5.20 & -5.20 \\
\hline Zolmitriptan & -4.48 & -4.32 & -6.32 & -6.01 & -5.56 & -5.56 & -6.38 & -5.7 \\
\hline
\end{tabular}


163 Table 2B. Minimum and most populated values, expressed as $\mathrm{kcal} \mathrm{mol}^{-1}$, of the cluster affinities of the analytes for the second four (from 5 to 8) discrete binding sites located on the P-gp.

\begin{tabular}{|c|c|c|c|c|c|c|c|c|}
\hline Analyte & $\begin{array}{l}\text { P-gp } \\
5 \text { Min }\end{array}$ & $\begin{array}{l}\text { P-gp } 5 \\
\text { MP }\end{array}$ & $\begin{array}{l}\text { P-gp } \\
\text { 6 Min }\end{array}$ & $\begin{array}{l}\text { P-gp } \\
6 \text { MP } \\
\end{array}$ & $\begin{array}{l}\text { P-gp } \\
7 \text { Min } \\
\end{array}$ & $\begin{array}{l}\text { P-gp } \\
7 \text { MP } \\
\end{array}$ & $\begin{array}{l}\text { P-gp } \\
8 \text { Min }\end{array}$ & $\begin{array}{l}\text { P-gp } \\
8 \text { MP }\end{array}$ \\
\hline 2-(Methylamino)pyridine & -3.03 & -3.03 & -3.40 & -3.40 & -3.53 & -3.53 & -3.22 & -3.22 \\
\hline 2,2,2-trifluoroethyl vinyl ether & -1.72 & -1.64 & -2.05 & -2.05 & -2.18 & -2.18 & -1.93 & -1.93 \\
\hline 2,6-diisopropylphenol & -4.42 & -4.22 & -5.17 & -5.17 & -5.56 & -5.56 & -4.67 & -4.67 \\
\hline Acetaminophen & -3.81 & -3.81 & -4.20 & -4.20 & -4.10 & -4.10 & -3.37 & -3.37 \\
\hline Acetylsalicylic acid & -3.86 & -3.86 & -3.91 & -3.91 & -4.42 & -4.42 & -3.68 & -3.68 \\
\hline Aminopyrine & -5.20 & -5.18 & -5.36 & -4.91 & -5.60 & -5.60 & -4.47 & -4.47 \\
\hline Amitriptyline & -6.00 & -5.92 & -6.85 & -6.85 & -7.49 & -7.49 & -5.63 & -5.63 \\
\hline Amobarbital & -4.51 & -4.51 & -4.40 & -4.05 & -4.99 & -4.99 & -4.23 & -4.23 \\
\hline Antipyrine & -4.78 & -4.78 & -4.77 & -4.77 & -5.58 & -5.58 & -4.31 & -4.30 \\
\hline Atenolol & -4.03 & -4.03 & -4.82 & -4.60 & -4.86 & -4.86 & -3.37 & -3.37 \\
\hline Benzene & -3.19 & -3.19 & -3.26 & -3.26 & -3.31 & -3.31 & -3.01 & -3.01 \\
\hline Betahistine & -2.93 & -2.93 & -3.54 & -3.54 & -3.52 & -3.52 & -3.41 & -3.41 \\
\hline Caffeine & -3.64 & -3.64 & -3.76 & -3.76 & -4.56 & -4.56 & -3.76 & -3.76 \\
\hline Carbamazepine & -5.87 & -5.87 & -6.16 & -6.16 & -6.83 & -6.83 & -5.31 & -5.31 \\
\hline Celecoxib & -5.93 & -5.31 & -5.82 & -5.82 & -7.97 & -7.97 & -5.59 & -5.04 \\
\hline Chlorambucil & -4.30 & -4.30 & -4.57 & -4.56 & -5.63 & -5.63 & -3.93 & -3.93 \\
\hline Chlorpromazine & -5.66 & -5.00 & -6.75 & -6.57 & -7.23 & -7.01 & -5.16 & -5.16 \\
\hline Cimetidine & -3.72 & -3.45 & -4.58 & -3.80 & -4.76 & -3.88 & -3.41 & -3.20 \\
\hline Citalopram & -5.63 & -5.59 & -5.85 & -5.69 & -6.44 & -6.44 & -4.93 & -4.86 \\
\hline Clonidine & -4.06 & -3.98 & -4.47 & -4.40 & -5.34 & -5.34 & -4.31 & -4.31 \\
\hline Clozapine & -5.44 & -5.18 & -6.64 & -6.64 & -7.05 & -7.05 & -4.98 & -4.95 \\
\hline Cotinine & -4.52 & -4.52 & -4.48 & -4.48 & -4.86 & -4.76 & -4.29 & -4.29 \\
\hline Cyclobenzaprine & -5.70 & -5.68 & -7.12 & -6.86 & -7.35 & -7.23 & -5.36 & -5.28 \\
\hline Desipramine & -5.74 & -5.74 & -6.44 & -6.44 & -6.42 & -5.73 & -4.78 & -4.78 \\
\hline Diclofenac & -5.23 & -5.23 & -5.96 & -5.46 & -6.32 & -6.32 & -4.65 & -4.37 \\
\hline Diphenhydramine & -5.07 & -5.07 & -5.41 & -4.78 & -5.42 & -5.42 & -3.89 & -3.54 \\
\hline Domperidone & -6.04 & -5.71 & -5.73 & -5.73 & -7.69 & -7.69 & -6.26 & -6.13 \\
\hline Donepezil & -7.11 & -6.10 & -7.07 & -7.07 & -7.83 & -7.77 & -6.58 & -6.58 \\
\hline Eserine & -5.52 & -5.50 & -5.57 & -5.57 & -5.92 & -5.92 & -4.57 & -4.54 \\
\hline Ethosuximide & -3.62 & -3.62 & -4.16 & -4.16 & -4.34 & -4.34 & -3.76 & -3.35 \\
\hline Ethylbenzene & -3.58 & -3.58 & -3.96 & -3.96 & -4.21 & -4.21 & -3.71 & -3.71 \\
\hline Fluphenazine & -4.39 & -4.39 & -5.23 & -3.06 & -6.46 & -5.16 & -5.79 & -5.79 \\
\hline Haloperidol & -5.68 & -5.14 & -5.35 & -4.41 & -7.32 & -7.31 & -5.48 & -5.35 \\
\hline Halothane & -2.37 & -2.37 & -2.57 & -2.55 & -2.86 & -2.86 & -2.41 & -2.35 \\
\hline Hexobarbital & -5.21 & -4.99 & -5.22 & -5.22 & -5.51 & -5.51 & -4.56 & -4.50 \\
\hline Hydroxyzine & -5.26 & -4.29 & -5.29 & -5.29 & -6.18 & -5.89 & -4.57 & -4.50 \\
\hline Ibuprofen & -4.84 & -4.84 & -4.91 & -4.69 & -5.41 & -5.41 & -4.55 & -4.55 \\
\hline Imipramine & -5.83 & -5.18 & -6.69 & -6.32 & -6.67 & -6.67 & -4.97 & -4.97 \\
\hline
\end{tabular}




\begin{tabular}{|c|c|c|c|c|c|c|c|c|}
\hline Indomethacin & -5.92 & -5.92 & -6.44 & -6.44 & -7.22 & -6.91 & -5.26 & -5.26 \\
\hline Ketorolac & -5.73 & -5.68 & -5.71 & -5.63 & -6.36 & -6.35 & -5.01 & -4.74 \\
\hline Lamotrigine & -4.13 & -4.09 & -4.77 & -4.77 & -5.30 & -5.23 & -4.32 & -3.66 \\
\hline Levofloxacin & -5.40 & -5.14 & -3.46 & -3.46 & -6.37 & -6.37 & -4.71 & -4.71 \\
\hline Metanol & -1.47 & -1.47 & -1.38 & -1.38 & -1.37 & -1.37 & -1.42 & -1.42 \\
\hline Metoclopramide & -3.63 & -3.36 & -4.40 & -4.02 & -4.95 & -4.95 & -3.55 & -2.80 \\
\hline Metoprolol & -3.49 & -3.46 & -4.25 & -4.25 & -4.60 & -4.39 & -3.57 & -3.42 \\
\hline Mianserin & -5.89 & -5.89 & -6.21 & -6.21 & -7.07 & -7.07 & -5.63 & -5.63 \\
\hline Naproxen & -5.26 & -5.26 & -5.48 & -5.48 & -5.80 & -5.80 & -4.72 & -4.71 \\
\hline Nicotine & -3.77 & -3.77 & -4.42 & -4.20 & -4.67 & -4.67 & -4.24 & -4.13 \\
\hline Nitrofurantoin & -4.37 & -4.30 & -4.62 & -4.31 & -5.24 & -5.24 & -3.90 & -3.67 \\
\hline Norfloxacin & -4.46 & -4.44 & -3.73 & -2.70 & -5.85 & -5.83 & -4.99 & -4.99 \\
\hline Nortriptyline & -5.98 & -5.98 & -7.20 & -7.20 & -7.09 & -7.07 & -5.30 & -5.05 \\
\hline Olanzapine & -5.42 & -5.42 & -6.24 & -6.24 & -6.48 & -6.48 & -5.06 & -5.06 \\
\hline Omeprazole & -5.24 & -5.22 & -6.47 & -6.47 & -7.26 & -6.79 & -5.28 & -4.22 \\
\hline Oxazepam & -5.96 & -5.94 & -6.61 & -6.61 & -6.81 & -6.70 & -5.02 & -5.02 \\
\hline Paroxetine & -5.71 & -5.03 & -6.14 & -4.23 & -7.49 & -6.50 & -4.97 & -4.97 \\
\hline Pentobarbital & -4.29 & -4.18 & -4.35 & -4.22 & -4.84 & -4.84 & -3.75 & -3.60 \\
\hline Phenylbutazone & -6.29 & -6.19 & -7.19 & -7.19 & -7.33 & -6.78 & -5.57 & -5.44 \\
\hline Phenytoin & -5.80 & -5.80 & -5.42 & -5.38 & -6.25 & -6.25 & -4.67 & -4.64 \\
\hline Pindolol & -3.61 & -3.58 & -5.72 & -5.72 & -5.29 & -5.13 & -4.03 & -3.81 \\
\hline Primidone & -4.43 & -4.31 & -4.99 & -4.99 & -5.23 & -5.23 & -4.26 & -4.08 \\
\hline Promazine & -5.47 & -4.86 & -6.22 & -5.94 & -6.47 & -6.05 & -4.71 & -4.50 \\
\hline Promethazine & -5.55 & -4.99 & -6.07 & -5.87 & -6.62 & -6.40 & -4.72 & -4.68 \\
\hline Propranolol & -4.42 & -4.42 & -5.99 & -5.19 & -5.67 & -5.62 & -4.30 & -4.30 \\
\hline Quinidine & -5.68 & -5.68 & -6.74 & -5.62 & -7.92 & -7.78 & -5.32 & -4.72 \\
\hline Ranitidine & -2.99 & -2.99 & -3.61 & -3.34 & -3.76 & -2.86 & -2.12 & -1.63 \\
\hline Rifampicin & -5.13 & -3.28 & -6.66 & -6.00 & -7.44 & -7.00 & -3.14 & -3.14 \\
\hline Ropinirole & -4.25 & -4.17 & -5.98 & -5.98 & -6.22 & -6.22 & -4.11 & -3.39 \\
\hline Salicylic acid & -3.09 & -3.09 & -3.42 & -3.42 & -3.85 & -3.85 & -3.31 & -3.31 \\
\hline Theobromine & -3.46 & -3.46 & -3.49 & -3.49 & -4.29 & -4.29 & -3.80 & -3.80 \\
\hline Theophylline & -3.63 & -3.63 & -3.72 & -3.72 & -4.45 & -4.45 & -3.66 & -3.66 \\
\hline Toluene & -3.42 & -3.42 & -3.79 & -3.79 & -3.93 & -3.93 & -3.39 & -3.39 \\
\hline Tramadol & -4.46 & -4.46 & -5.36 & -5.36 & -5.62 & -5.46 & -4.26 & -4.26 \\
\hline Trazodone & -6.06 & -6.26 & -6.86 & -6.86 & -7.40 & -7.29 & -6.06 & -5.47 \\
\hline Triprolidine & -6.48 & -6.48 & -6.40 & -5.53 & -7.28 & -7.28 & -5.38 & -5.26 \\
\hline Valproic acid & -3.03 & -3.03 & -3.10 & -3.10 & -3.76 & -3.76 & -2.90 & -2.90 \\
\hline Venlafaxine & -4.64 & -4.33 & -5.60 & -5.23 & -6.48 & -6.48 & -4.40 & -4.40 \\
\hline Verapamil & -5.16 & -4.84 & -4.74 & -3.51 & -6.80 & -6.53 & -4.32 & -3.72 \\
\hline Zidovudine & -3.94 & -3.93 & -4.49 & -4.49 & -4.55 & -4.55 & -3.90 & -3.19 \\
\hline Zolmitriptan & -4.78 & -3.70 & -6.13 & -6.13 & -6.15 & -6.15 & -4.42 & -4.42 \\
\hline
\end{tabular}




\subsection{MLC INDEXES IN LOG BB PREDICTION}

MLC indexes were used to develop BBB passage potential predicting models along with either static or conformational properties. At first, all the analytes were assumed as having zero atomic charges, even the ones supporting one or more ionizable functions. The equations along with the statistical validation are reported in Table 3 . In the equations thereby reported, $r^{2}$ is the multiple regression coefficient, $q^{2}$ is the $r^{2}$ validated by Leave-One-Out (LOO) optimization, $S E$ is the error standard deviation, $F$ represents the Fischer regression statistical value, $P C$ is the Amemiya predictive criterion and ExRow is the analyte excluded for maximizing the predictive strength of the statistical model. If not differently indicated, every regression was developed by employing four different independent variables (MLC indexes + three other physico-chemical descriptors). Surprisingly, even if over two thirds of the analytes support one or more ionizable functions, fairly good relationships, as the one expressed by equations (1) and (2), are obtained even not taking into account the presence of electric charges. This may be attributed to the fact that, although the molecular mechanisms involved in MLC are multiple and complex, the occurrence of analyte/micelles electrostatic interactions plays a pivotal role in the global retention and it appears reasonable to assume that such interactions are encoded in MLC indexes. It should be also highlighted that, in these specific cases, being VirtualLogP values calculated starting from the analytes assumed in their forms having zero atomic charges, such values can be reasonably assumed as estimates of their $\log \mathrm{P}^{\mathrm{N}}$ values. Subsequently, the analytes supporting extensively ionizable functions (i.e. carboxy groups, for acids primary, secondary and tertiary amines for bases) were assumed as completely charged, regardless of the relative abundance of the charged species at the physiological $\mathrm{pH}$. Considering the ionizable analytes as entirely charged species slightly worsened the relationships (equations (3) and (4)). It should be pointed out that verapamil, the analyte excluded to maximize the predictive strength of the statistical model is a well-known P-gp substrate $^{26}$. P-gp is an ATP-dependent efflux pump, with broad substrate specificity which pumps many foreign substances out of cells ${ }^{27}$. Although it is widely expressed in the intestinal epithelium, 
liver cells and proximal tubule of the kidney, P-gp is also localized in the capillary endothelial cells composing the $\mathrm{BBB}$ and is responsible, for some classes of actives, of multi-drug resistance. Eventually, a weighted average of the static properties at physiological $\mathrm{pH}$ (7.4), according to the pKa of each compound, was performed. For zwitterions, the static properties were calculated for each microspecies possibly present at $\mathrm{pH} 7.4$ and their relative abundances, calculated by the software Marvin Sketch 15.1 for Mac OS $\mathrm{X}^{28}$, were also used to perform the weighted averages. The relative abundances of the microspecies present at $\mathrm{pH} 7.4$ are reported in the Supporting Information section for the ampholytes levofloxacin (page S-2), norfloxacin (page S-4) and omeprazole (page S-6). This approach was adapted to mirror more closely what actually occurs in vivo. Performing the weighted average of the properties benefited noticeably the relationships as described by equations (5) and (6). It is also interesting to note how, according to the above reported relationships, the $\mathrm{BBB}$ penetration of drugs will be enhanced for highly retained compounds in MLC, how it is hindered by the occurrence of drug/membrane polar (Psa)/ electrostatic (Dipole) interactions, and how the transport through the barrier seems favored for bases (Charge). However, by taking into account the analytes assumed as static, the properties are derived considering them in their minimum energy conformations, i.e. after minimization. Indeed, several authors ${ }^{29}$ reported that such conformations are not always the ones actually involved in membrane barrier passage. Therefore, a conformational analysis in vacuum was carried out for each analyte included in the data set by using the Boltzmann Jump method that generates at random 1000 possible conformations by exploring the conformational space of the rotatable dihedral angles. The conformational analysis was first performed on the analytes assumed as having zero atomic charges, then on the analytes assumed as completely charged and finally taking into account a weighted average of the properties at the experimental $\mathrm{pH} 7.4$, according to the $\mathrm{pKa}$ of each analyte. In the following models the conformational properties were considered separately to look into the predictive strength of the models. As it is evident from Table 3, the use of conformational properties instead of the static ones slightly worsened the relationships. This aspect is quite 
219 interesting as the calculation of conformational properties can be rather time-consuming especially 220 if the compound libraries to screen are wide and the computers employed are not sufficiently 221 powerful. Conversely, the static properties are much faster to calculate. Performing the weighted 222 average of the conformational properties yielded the most predictive models (equations (11) and 223 (12)) and in those relationships, verapamil again behaved as an outlier, suggesting that such models 224 would not be able to mirror the penetration of analytes undergoing some sort of active transport, in 225 this case P-gp mediated efflux. It is interesting to point out how, among the ionized properties 226 employed for the statistical method development (equations (9) and (10)), no one depends 227 noticeably on ionization. Furthermore, the conformational analysis demonstrated how it is the PSA 228 Max, i.e. the maximum value that the PSA assumes by exploring the conformational space in 229 vacuum of each analyte, that best relates with $\log \mathrm{BB}$ values as those values are incorporated in 230 each model based on conformational properties (equations (7), (8), (9), (10), (11) and (12)). 
Table 3. Statistical validation of the models developed employing $\log \mathrm{k}_{\mathrm{w}}$ SDS values of the dataset $(n=79)$ along with three other physico-chemical descriptors.

\begin{tabular}{|c|c|c|c|c|c|c|c|c|c|c|c|c|}
\hline $\begin{array}{l}\text { MOLECULAR } \\
\text { DESCRIPTORS }\end{array}$ & $r^{2}$ & $q^{2}$ & $S E$ & $F$ & $P C$ & $\begin{array}{l}r^{2} \\
(\mathrm{n}- \\
1)\end{array}$ & $\begin{array}{l}S E \\
(\mathrm{n}-1)\end{array}$ & $\begin{array}{l}F(\mathrm{n}- \\
1)\end{array}$ & $\begin{array}{l}P C(\mathrm{n}- \\
\text { 1) }\end{array}$ & EX-ROW & EQUATIONS & $\begin{array}{l}\text { EQ } \\
\text { No }\end{array}$ \\
\hline \multicolumn{13}{|l|}{ STATIC } \\
\hline $\begin{array}{l}\text { ZERO ATOMIC } \\
\text { CHARGES } \\
\text { PROPERTIES }\end{array}$ & 0.69 & 0.65 & 0.521 & 41.42 & 21.656 & 0.71 & 0.510 & 44.06 & 20.535 & $\begin{array}{l}- \\
\text { - } \\
\text { (Methylamino) } \\
\text { pyridine }\end{array}$ & $\begin{array}{l}\log \mathrm{BB}=- \\
0.2693+0.8191 \\
\log \mathrm{k}_{\mathrm{w}}{ }^{\mathrm{sDS}} \text { - } \\
0.0162 \text { Psa - } \\
0.0824 \\
\text { VirtualLogP + } \\
0.1456 \mathrm{HbDon} \\
\log \mathrm{BB}=- \\
0.2166+0.8383 \\
\log \mathrm{k}_{\mathrm{w}}{ }^{\mathrm{SDS}} \text { - } \\
0.0170 \text { Psa - } \\
0.0994 \\
\text { VirtualLogP + } \\
0.1570 \mathrm{HbDon}\end{array}$ & 2 \\
\hline $\begin{array}{l}\text { IONIZED } \\
\text { PROPERTIES }\end{array}$ & 0.68 & 0.63 & 0.530 & 39.28 & 22.452 & 0.70 & 0.517 & 42.20 & 21.104 & Verapamil & $\begin{array}{l}\log \mathrm{BB}=- \\
0.3460+0.6671 \\
\log \mathrm{k}_{\mathrm{w}}{ }^{\text {} D S}- \\
0.0104 \text { Psa }+ \\
0.1425 \text { Charge - } \\
0.0138 \\
\text { Impropers } \\
\\
\log \mathrm{BB}=- \\
0.4024+0.7071 \\
\log \mathrm{k}_{\mathrm{w}} \text { sDs - } \\
0.0093 \text { Psa }+ \\
0.1297 \text { Charge - } \\
0.0187 \\
\text { Impropers }\end{array}$ & 3 \\
\hline $\begin{array}{l}\text { WEIGHTED } \\
\text { AVERAGE }\end{array}$ & 0.72 & 0.68 & 0.498 & 47.05 & 19.795 & 0.73 & 0.486 & 50.29 & 18.617 & - & $\begin{array}{l}\log \mathrm{BB}=- \\
0.3145+0.6825 \\
\log \mathrm{k}_{\mathrm{w}}{ }^{\text {SDS }}- \\
0.0091 \text { Psa - } \\
0.0202 \text { Dipole + } \\
0.2042 \text { Charge } \\
\\
\log \mathrm{BB}=- \\
0.3145+0.6825 \\
\log \mathrm{k}_{\mathrm{w}}{ }^{\text {sDs }} \\
0.0091 \text { Psa - }\end{array}$ & 5 \\
\hline
\end{tabular}




\begin{tabular}{|c|c|c|c|c|c|c|c|c|c|c|c|c|}
\hline & & & & & & & & & & & $\begin{array}{l}0.0202 \text { Dipole }+ \\
0.2042 \text { Charge }\end{array}$ & \\
\hline \multicolumn{13}{|c|}{ CONFORMATIONAL } \\
\hline $\begin{array}{l}\text { ZERO ATOMIC } \\
\text { CHARGES } \\
\text { PROPERTIES }\end{array}$ & 0.69 & 0.64 & 0.526 & 40.25 & 22.081 & 0.71 & 0.509 & 44.37 & 20.426 & - & $\begin{array}{l}\log \mathrm{BB}=- \\
0.4857+0.8201 \\
\log \mathrm{k}_{\mathrm{w}}{ }^{\mathrm{DDS}}- \\
0.0044 \text { PSA } \\
\text { Max - 0.0708 } \\
\text { MD Max - } \\
0.2671 \mathrm{MD} \text { sd } \\
\log \mathrm{BB}=- \\
0.4973+0.8307 \\
\log \mathrm{k}_{\mathrm{w}}{ }^{\mathrm{DDS}} \text { - } \\
0.0048 \text { PSA } \\
\text { Max - 0.0583 } \\
\text { MD Max - } \\
0.3274 \text { MD sd }\end{array}$ & 7 \\
\hline $\begin{array}{l}\text { IONIZED } \\
\text { PROPERTIES }\end{array}$ & 0.65 & 0.62 & 0.549 & 69.77 & 23.504 & 0.67 & 0.538 & 74.60 & 22.304 & Primidone & $\begin{array}{l}\log \mathrm{BB}=- \\
0.5392+0.7898 \\
\log \mathrm{k}_{\mathrm{w}}{ }^{\mathrm{DDS}} \text { - } \\
0.0093 \text { PSA } \\
\operatorname{Max} \\
\log \mathrm{BB}=- \\
0.5338+0.8008 \\
\log \mathrm{k}_{\mathrm{w}}{ }^{\text {DD }} \text { - } \\
0.0099 \text { PSA } \\
\text { Max }\end{array}$ & 9 \\
\hline $\begin{array}{l}\text { WEIGHTED } \\
\text { AVERAGE }\end{array}$ & 0.68 & 0.63 & 0.534 & 38.57 & 22.729 & 0.70 & 0.520 & 41.69 & 21.283 & - & $\begin{array}{l}\log \mathrm{BB}=- \\
0.4329+0.7969 \\
\log \mathrm{k}_{\mathrm{w}} \mathrm{sD}- \\
0.0072 \mathrm{PSA} \\
\text { Max - } 0.0235 \\
\text { MD Min - } \\
0.0485 \mathrm{MLP} \\
\text { Average } \\
\\
\\
\log \mathrm{BB}=- \\
0.4911+0.8121 \\
\log \mathrm{k}_{\mathrm{w}} \mathrm{SDS}- \\
0.0068 \text { PSA } \\
\text { Max - } 0.0233 \\
\text { MD Min - } \\
0.0334 \text { MLP } \\
\text { Average }\end{array}$ & 11 \\
\hline
\end{tabular}

236 The same approach was extended to the IAM indexes. The equations along with the statistical 237 validation coefficient are reported in Table 4. Indeed, taking into account either the properties of the analytes assumed as having zero atomic charges (equations (13) and (14)) or those of the analytes 
assumed as completely charged (equations (15) and (16)) resulted in a BBB passage predictive

240 strength inferior to that obtained by using MLC indexes. Such conclusions are supported by the 241 lower correlation coefficients obtained. It is interesting to note how domperidone, the compound 242 excluded in first best optimized model described by equation (14), is a well-known substrate of the $243 \mathrm{P}-\mathrm{gp}^{26}$, and is pumped out of cells by such efflux system despite its high biomembrane passive 244 diffusion. Analogously to what was observed in the analysis of MLC indexes, performing the 245 weighted average of the static properties resulted the winning strategy also for this set of 246 experimental measures. In fact, a $0.72 r^{2}(n-1)$, achieved on a set as large as 79 analytes, employing 247 only four descriptors suggests that the model (equations (17) and (18)) is robust and reliable 248 However, these relationships are roughly comparable to those obtained by using MLC indexes 249 (equations (5) and (6)). This evidence is indeed rather surprising, since the IAM stationary phase 250 consists of analogues of phosphatidylcholine, the most abundant phospholipid expressed in the 251 capillary endothelium acting as a barrier between the blood and the cerebrospinal fluids (CSF), and thus they would represent an ideal biomimetic system. Conversely, this kind of SDS based MLC should have drawbacks arising from the different chemical structure of SDS in comparison with membrane phospholipids. But this evidence would suggest that they are incidentally able to mirror 255 the drug/membrane interactions involved in vivo thanks to the peculiar amphiphilic features of the anionic micelles. In fact, for some reasons, they seem to be able to model the passive BBB penetration of drugs fairly better than IAM indexes. Furthermore, the physico-chemical descriptors reported in equation (18) are the same as the ones in equation (6), supporting again the concept according to which the polar (Psa) /electrostatic (Dipole) interaction component plays a relevant 260 role in hindering the BBB penetration of drugs. Again, bases seem to be favored in BBB entering and this is also consistent with the clinical experience. In fact, polar and extensively protonated at pH 7.4 basic compounds, such as amphetamine and methamphetamine, are known to have an appreciable CNS activity but it is much harder to recall similar cases for polar acids. The conformational analyses of the analytes neither assumed as having zero atomic charges, nor as 
265 ionized benefitted the relationships. Moreover, even performing the weighted average was not 266 beneficial at all for the relationships (data not shown). 
Table 4. Statistical validation of the models developed employing $\log \mathrm{k}_{30 \% \mathrm{MeOH}}{ }^{\text {IAM }}$ values of the dataset $(n=79)$ along with three other physico-chemical descriptors.

270

\begin{tabular}{|c|c|c|c|c|c|c|c|c|c|c|c|c|}
\hline $\begin{array}{l}\text { MOLECULAR } \\
\text { DESCRIPTORS }\end{array}$ & $r^{2}$ & $q^{2}$ & $S E$ & $F$ & $P C$ & $\begin{array}{l}r^{2} \\
(\mathrm{n}- \\
1)\end{array}$ & $\begin{array}{l}S E \\
(\mathrm{n}-1)\end{array}$ & $\begin{array}{l}F(\mathrm{n}- \\
1)\end{array}$ & $\begin{array}{l}P C(\mathrm{n}- \\
1)\end{array}$ & EX-ROW & EQUATIONS & $\begin{array}{l}\text { EQ } \\
\text { No }\end{array}$ \\
\hline \multicolumn{13}{|l|}{ STATIC } \\
\hline $\begin{array}{l}\text { ZERO ATOMIC } \\
\text { CHARGES } \\
\text { PROPERTIES }\end{array}$ & 0.64 & 0.59 & 0.561 & 33.08 & 25.156 & 0.67 & 0.540 & 36.88 & 23.025 & $\begin{array}{l}- \\
\text { Domperidone }\end{array}$ & $\begin{array}{l}\log \mathrm{BB}=+0.6691+ \\
0.8369 \log \mathrm{k}_{30 \%} \\
\text { MeOH }^{\text {IAM }}-0.0166 \text { Psa }- \\
0.1473 \text { VirtualLogP }+ \\
0.1139 \text { HbDon } \\
\log \mathrm{BB}=+0.6706+ \\
0.9057 \log \mathrm{k}_{30 \%} \\
\text { MeOH }^{\text {IAM }}-0.0161 \text { Psa - } \\
0.1473 \text { VirtualLogP }+ \\
0.1173 \text { HbDon }\end{array}$ & 13 \\
\hline $\begin{array}{l}\text { IONIZED } \\
\text { PROPERTIES }\end{array}$ & 0.64 & 0.58 & 0.561 & 33.07 & 25.155 & 0.67 & 0.540 & 37.29 & 22.976 & $\begin{array}{l}- \\
\text { Lamotrigine }\end{array}$ & 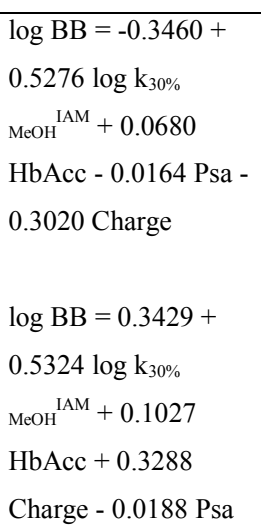 & 15 \\
\hline $\begin{array}{l}\text { WEIGHTED } \\
\text { AVERAGE }\end{array}$ & 0.70 & 0.65 & 0.515 & 42.79 & 21.169 & 0.72 & 0.494 & 47.40 & 19.219 & $\begin{array}{l}- \\
\text { Celecoxib }\end{array}$ & $\begin{array}{l}\log \mathrm{BB}=+0.4388+ \\
0.5458 \log \mathrm{k}_{30 \%} \\
{ }_{\mathrm{MeOH}}{ }^{\mathrm{AM}}-0.0110 \text { Psa }- \\
0.0190 \text { Dipole }+ \\
0.4653 \text { Charge } \\
\text { log BB }=+0.3773+ \\
0.6063 \log \mathrm{k}_{30 \%} \\
{ }_{\text {IAM }} \text { - } 0.0097 \text { Psa - } \\
0.0207 \text { Dipole }+ \\
0.4182 \text { Charge }\end{array}$ & 17 \\
\hline
\end{tabular}




\subsection{IAM + MLC INDEXES IN LOG BB PREDICTION}

273 In the present study, MLC and IAM indexes were, in a first instance, considered separately. 274 However, the evident differences in the elution order observed depict a rather different selectivity 275 between both techniques. For this reason, the development of the BBB entering potential statistical 276 models was also performed by considering both the chromatographic indexes at the same time, 277 along with three other molecular descriptors (five independent variables in total), starting from the 278 weighted average of the molecular properties. This strategy resulted in a markedly improved 279 predictive strength (equations (19) and (20)) as reported in Table 5. These relationships may 280 suggest that the molecular mechanism involved in IAM-LC and MLC are different but play both a 281 relevant role in BBB diffusion of drugs.

\subsection{P-GP AFFINITIES IN LOG BB PREDICTION}

283 As already mentioned, each analyte present in the dataset was docked into each discrete binding site 284 on the P-gp and the binding affinities were incorporated in the development of BBB passage predictive statistical models. Indeed, recent fuctional studies have identified seven sometimes overlapping binding sites accommodating substrates and inhibitors in the greasy, polyspecific binding cavity of P-gp. These binding sites were demonstrated able to allosterically communicate in a negative heterotropic manner. Moreover, an additional binding site was recognized on the exterior of P-gp bounded by residues from the transmembrane helices 9, 12 and the elbow helix 2. This site faces away from the transporter, lying close to the predicted membrane-water interface and intramembranous substrate-entry portal.

292 On average, highly clustered results were achieved, meaning that the conformational search procedure was exhaustive enough to ensure a coverage of the accessible conformational space. An extensive cluster analysis (RMSD tolerance was set to $2.0 \AA$ ) was performed and the binding affinity now reported in Table $2 \mathrm{~A}$ and Table $2 \mathrm{~B}$ are the minimum and the most populated binding 
297 than $\pm 1.8 \mathrm{kcal} \mathrm{mol}^{-1}$. However, from the relationships reported above, P-gp affinities do not seem 298 to have an appreciable role in BBB passage. However, this is not entirely true because the statistical 299 model development was carried out using only four independent variables, thus leading the 300 software to select only the four most relevant descriptors, among which P-gp affinities were not 301 included. Indeed, when five independent variables were set in the statistical method development, 302 the P-gp binding affinities (Table 5A and 5B) were used by the software to build up the models. 303 Equations (21) and (22), generated by IAM indexes and four static properties of the analytes, 304 assumed as having zero atomic charges, is an example as can be seen in Table 5. The AutoDock 305 GPF/DPF files for site 1 and 7, i.e. the ones actually involved in the statistical models (21) and (22), 306 are now provided as supplementary materials.

This is not surprising because among the considered analytes, the only ones known from the 308 literature to be substrates of P-gp are cimetidine, domperidone, ranitidine, rifampicin, quinidine and 309 verapamil ${ }^{26}$, and they represent less than $5 \%$ of the dataset. Indeed, the compounds considered were 310 selected in the attempt to mirror as accurately and completely as possible the marketed drugs, in 311 terms of diverse chemical nature, molecular volume, CNS activity and molecular lipophilicity. 312 Since the active transport comprises only for a minority of drugs, whereby the drug uptake in 313 mainly driven by passive transcellular diffusion, the limited predictivity of the P-gp molecular 314 affinity may be dataset related. This approach suffers from some limitations, the most evident one 315 being the aspect that the receptor flexibility is not taken into account. The main reason behind it is 316 the large number of degrees of freedom that should be considered in this kind of calculations, thus 317 requiring remarkable computational power. However, neglecting the receptor flexibility could lead 318 to poor docking results in terms of binding pose prediction in real-world settings. Therefore, these 319 results must be regarded as a preliminary attempt to gain new insights and model the active efflux 320 of drugs pumped out of cells by P-gp, being neither exhaustive nor complete. Other experiments 321 have to be performed and docking conditions further calibrated in order to validate the proposed 
322 model.

323 
324 Table 5. Statistical validation of the models developed employing either $\log \mathrm{k}_{\mathrm{w}}{ }^{\mathrm{SDS}}$ or $\log \mathrm{k}_{30} \% \mathrm{MeOH}$ IAM values of the dataset ( $n=79)$ along with four (equations (29-32)) or five (equations (25-28))

\begin{tabular}{|c|c|c|c|c|c|c|c|c|c|c|c|c|}
\hline STATIC & $r^{2}$ & $q^{2}$ & $S E$ & $F$ & $P C$ & $\begin{array}{l}r^{2}(\mathrm{n}- \\
1)\end{array}$ & $\begin{array}{l}S E(\mathrm{n}- \\
1)\end{array}$ & $\begin{array}{l}F(\mathrm{n}- \\
1)\end{array}$ & $\begin{array}{l}P C(\mathrm{n}- \\
1)\end{array}$ & EX-ROW & EQUATIONS & $\begin{array}{l}\text { EQ } \\
\text { No }\end{array}$ \\
\hline MLC + IAM & 0.74 & 0.69 & 0.477 & 42.45 & 18.409 & 0.77 & 0.454 & 48.09 & 16.447 & Domperidone & 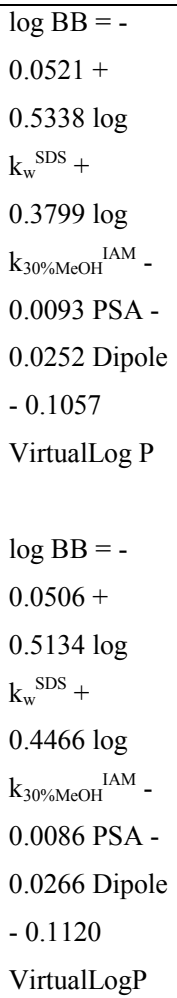 & 19 \\
\hline $\begin{array}{l}\text { P-GP AFFINITIES + } \\
\text { IAM }\end{array}$ & 0.67 & 0.61 & 0.544 & 29.30 & 23.925 & 0.69 & 0.521 & 32.50 & 21.653 & $\begin{array}{r}- \\
-\end{array}$ & $\begin{array}{l}\log \text { BB }= \\
0.8837+ \\
0.9206 \log \mathrm{k} \\
\text { IAM - } 0.0131 \\
\text { Psa - } 0.2781 \\
\text { P-gp 1 Min - } \\
0.1399 \\
\text { VirtualLogP } \\
+0.2742 \text { P-gp } \\
7 \text { MP } \\
\text { log BB = } \\
0.8118+ \\
0.9239 \log \\
\mathrm{k}_{30 \%} \% \text { MoOH } \\
0.0135 \text { Psa - } \\
0.1574 \\
\text { VirtualLogP - } \\
0.2825 \text { P-gp } 1 \\
\text { Min + } 0.2575\end{array}$ & 21 \\
\hline
\end{tabular}




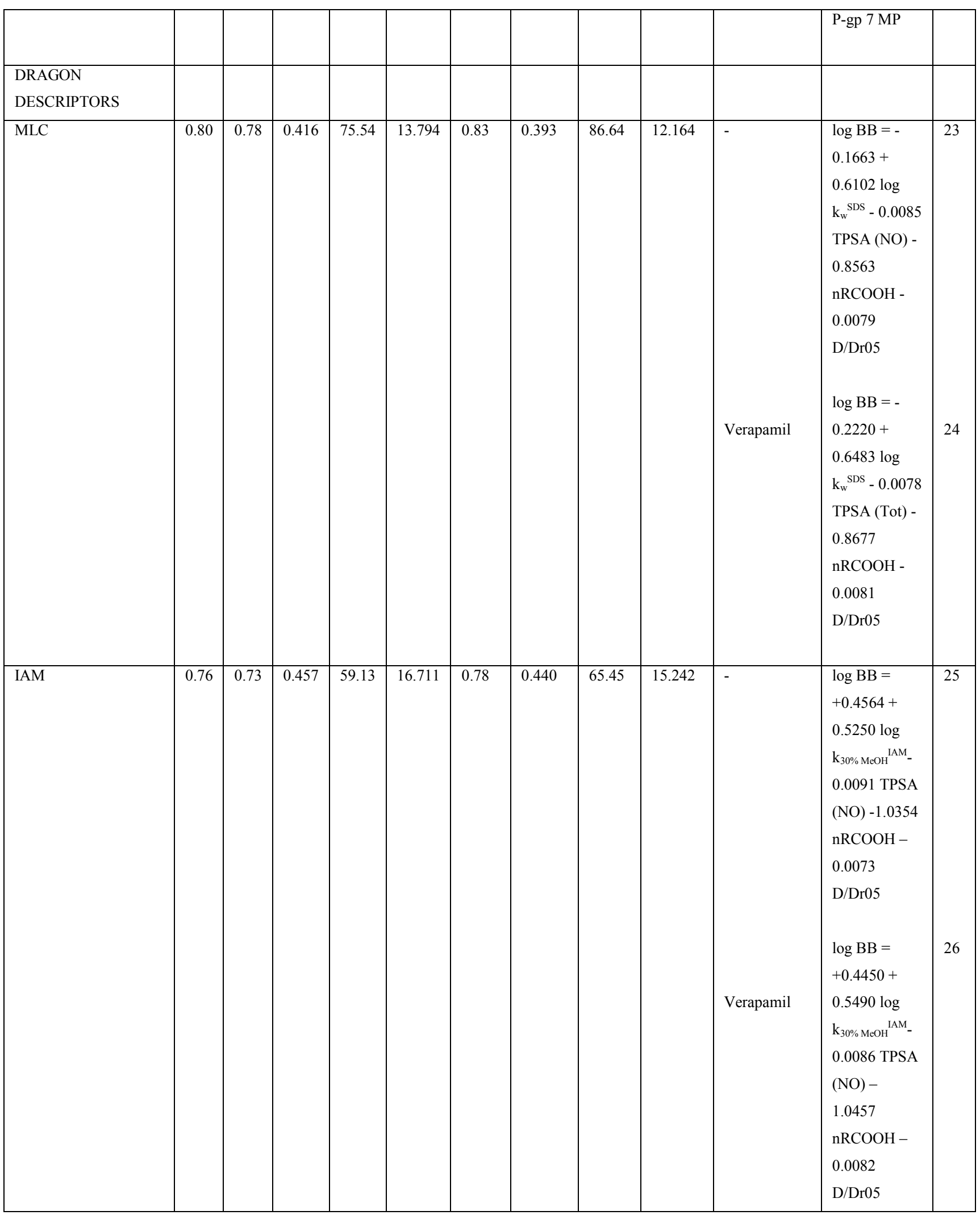



MODELS

334 In an attempt to further maximize the predictive strength of the models, IAM and MLC indexes were used in combination with E-Dragon descriptors ${ }^{30}$. The E-Dragon software calculates more than 1,600 descriptors, including not only the simplest atom type, functional group and fragment counts, but also several topological and geometrical descriptors. The results and statistical method validation are reported in Table 5. Remarkably high correlation coefficients were achieved with 339 either IAM $\left(r^{2}=0.78\right.$, equation (26)) or MLC $\left(r^{2}=0.83\right.$, equation (24)). As suggested by the 340 similarly high $q^{2}$ values, those relationships are not affected by any over fitting. The plots of the experimental vs predicted log BB values (as predicted by equation (24)) are reported in Figure 3. Such relationship is based on MLC indexes, TPSA (Tot) i.e. the topological polar surface area using nitrogen, oxygen, sulphur, phosphorus polar contributions which differs from the TPSA (NO), 344 included, for instance in equations (23), (25) and (26) that instead takes into account, in the topological polar surface area computation the nitrogen and oxygen contributions only. $\mathrm{nRCOOH}$ and D/Dr 05 are included in all the equations reported in Table 5. While the former is a functional group descriptor referring to the number of aliphatic carboxylic acids, the latter is a topological descriptor, named distance/detour ring index of order 5. It is based on operation over the distance/detour matrix $\mathrm{D} / \Delta$, a square symmetric matrix that contains the ratios of the lengths of the shortest to the longest path between any pair of vertices. It is calculated by the following equation:

$$
\mathrm{D} / \Delta=\frac{1}{2} \sum_{i=l}^{A} \sum_{j=l}^{A}(D / \Delta)_{i j}
$$

352 Although the role that such a parameter could play in the BBB partition is unclear, being its 353 interpretation quite difficult, it cannot be excluded that it might affect the molecular flexibility of 354 the analytes. However, the models obtained starting from E-Dragon descriptors would again 355 support the view according to which the BBB penetration of drugs would be enhanced for highly 
retained compounds either in IAM or MLC and hindered for compounds having greater PSA and

357 supporting one or even more acidic functions. To further validate the proposed method, the datasets 358 were divided randomly into 16 pairs of training and test sets. For each pair, the multiple linear 359 regression was performed and the equations derived from the training sets were subsequently used 360 to predict the $\log \mathrm{BB}$ values of the test sets. Such value set was used to evaluate the regression 361 coefficient $\left(r^{2}\right)$, the standard error (SE) of the estimates and the Fischer coefficients. The results of this additional validation are shown in Table 6.

Table 6. Validation of the best model employing four descriptors for log BB prediction.

\begin{tabular}{|c|c|l|l|c|c|}
\hline \multicolumn{7}{|c|}{ Model Validation } \\
\hline \multirow{2}{*}{ Trial } & \multicolumn{3}{|c|}{ Training set } & \multicolumn{2}{c|}{ Test set } \\
\cline { 2 - 6 } & $\mathbf{r}^{2}$ & SE & \multicolumn{1}{c|}{ F } & $\mathbf{r}^{2}$ & SE \\
\hline 1 & 0.87 & 0.320 & 57.459 & 0.75 & 0.496 \\
\hline 2 & 0.85 & 0.412 & 46.645 & 0.71 & 0.445 \\
\hline 3 & 0.84 & 0.411 & 45.880 & 0.71 & 0.498 \\
\hline 4 & 0.84 & 0.390 & 45.696 & 0.74 & 0.488 \\
\hline 5 & 0.84 & 0.415 & 43.969 & 0.75 & 0.433 \\
\hline 6 & 0.84 & 0.394 & 43.432 & 0.75 & 0.450 \\
\hline 7 & 0.83 & 0.389 & 41.407 & 0.77 & 0.455 \\
\hline 8 & 0.81 & 0.427 & 35.093 & 0.76 & 0.445 \\
\hline 9 & 0.80 & 0.444 & 35.085 & 0.78 & 0.411 \\
\hline 10 & 0.80 & 0.438 & 34.173 & 0.77 & 0.430 \\
\hline 11 & 0.80 & 0.426 & 33.984 & 0.79 & 0.453 \\
\hline 12 & 0.80 & 0.482 & 33.303 & 0.79 & 0.372 \\
\hline 13 & 0.80 & 0.444 & 33.181 & 0.78 & 0.424 \\
\hline 14 & 0.79 & 0.444 & 32.284 & 0.79 & 0.413 \\
\hline 15 & 0.77 & 0.462 & 28.660 & 0.81 & 0.404 \\
\hline 16 & 0.75 & 0.476 & 25.656 & 0.82 & 0.393 \\
\hline
\end{tabular}


370

371

372

373

374

375

376

377
Figure 3. Experimental vs Predicted log BB values plot for the best model obtained in the present study (Eq. (24)).

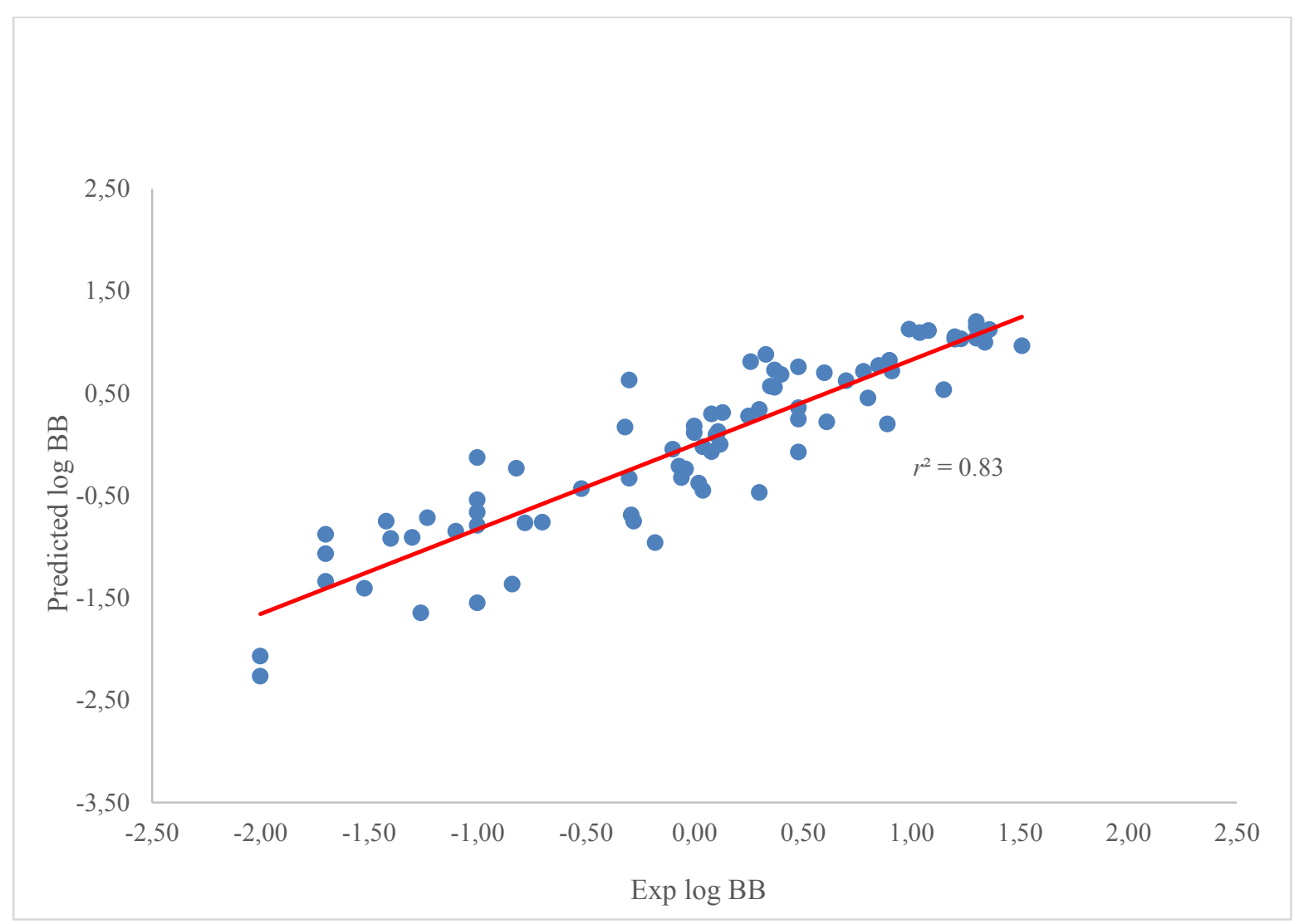




\subsection{CONCLUSION}

Highly significant $\left(r^{2}(n-1)\right.$ up to 0.83$)$ statistical methods for the BBB entering potential of drugs were achieved by applying the proposed method, which incidentally shed new light into BBB penetration of drugs. In fact, the $\mathrm{BBB}$ passage was found related to the analyte charges, being hindered for compounds supporting one or more acidic functions, and enhanced for bases. Moreover, molecules with higher dipolar momentum and greater PSA seemed less prone to cross the BBB. The relatively high number of analytes taken into account support statistically the suitability of the method as early screening method to evaluate BBB passage, and consolidate the novelty of the present work. In the modeling of drugs' BBB passage, both IAM and MLC indexes are found advantageously suitable; however, their combination with physico-chemical descriptors is highly beneficial for prediction. From a theoretical point of view, it should be considered that both IAM and MLC indexes relate to BBB passage data despite the different interactions they depict as confirmed by the lack of co-linearity between those two analytical indexes. Again, their simultaneous use in the statistic models, here performed for the first time, improved their prediction strength, thus suggesting that both play a relevant role in BBB passage although mirroring different phenomena. The P-gp efflux has also been investigated, but our results indicate that it would affect the overall BBB drug uptake only negligibly. However, this conclusion should be regarded cautiously due to the aspect that only a fewP-gp substrates were included in the set of analytes considered. Furthermore, the molecular docking simulations suffer from several limitations, the most important being the aspect that the receptor flexibility is not taken into account. This might have played a role in the moderate predictivity of the in silico calculated P-gp binding affinities. Finally, the proposed method is also suitable for pharmaceutical companies in the search for accurate $\mathrm{BBB}$ penetration oriented screening methods as the chromatographic conditions were carefully studied to obtain the indexes in a relatively short time such as to meet their demands. Chromatographic indexes (MLC and IAM) were always included in the best statistical models; this 
404 implies that the information encoded in such measures is original and cannot be satisfactorily 405 surrogated by other in silico descriptors. The molecular modeling performed was simple, easy-to406 perform and can be configured to run automatically in case of batch analyses. Furthermore, as the 407 method is rather cheap and relies on basic HPLC equipment, it offers potential for broad scale 408 application

\subsection{EXPERIMENTAL SECTION}

\subsection{CHEMICALS}

The solutes were obtained from Sigma-Aldrich (Machelen, Belgium), TCI-Europe (Zwijndrecht, 414 Belgium) and Thermofisher Acros Organics (Geel, Belgium) as listed in Table 1 and their purity was equal to or higher than $98 \%$.

\subsection{ANALYTICAL COLUMNS}

MLC and IAM experiments were performed on an Agilent Zorbax SB-C18 Rapid Resolution (3.5 $\mu \mathrm{m}, 50 \mathrm{~mm} \times 2.1 \mathrm{~mm}$; Santa Clara, CA, USA) and Regis IAM Fast Mini Screening (10 $\mu \mathrm{m}, 10 \mathrm{~mm}$ $\times 3.0 \mathrm{~mm}$; Morton Grove, IL, USA) columns, respectively.

424 MLC chromatographic analysis was performed on an Alliance, Waters 2690 chromatograph 425 (Milford, MA, USA) with a quaternary pump and an automatic injector. A Waters 2487 dualwavelength absorbance ultraviolet detector was used. The applied detection wavelengths for the

427 various solutes were always in the range between 210 and $300 \mathrm{~nm}$ as listed in Table 1. Data 
acquisition and processing were performed using a PeakSimple Chromatography Data System (model 202) and PeakSimple software (SRI Instruments, Torrance, CA, USA). The temperature of the analysis was controlled by a Polaratherm series 9000 unit (Selerity Technologies, Salt Lake City, USA) and set at $37^{\circ} \mathrm{C}$. The flow rate was $1.0 \mathrm{~mL} \mathrm{~min}^{-1}$ and the injection volume was $20 \mu \mathrm{L}$.

\subsubsection{IAM-HPLC}

IAM based chromatographic analysis was performed on an Agilent Capillary 1200 system (Santa Clara, CA, USA). The system included a capillary pump, a micro vacuum degasser and an automatic injector. An Agilent 1200 Series variable wavelength detector was used and set at the maximum absorbance wavelength of each analyte. The IAM-HPLC experiments were carried out at room temperature $\left(20 \pm 2{ }^{\circ} \mathrm{C}\right)$, the flow rate was $300 \mu \mathrm{L} \mathrm{min}{ }^{-1}$ and the injection volume was $1 \mu \mathrm{L}$.

\subsection{MOBILE PHASE AND SAMPLE PREPARATION}

MLC mobile phases were composed of aqueous solutions of $0.05 \mathrm{~mol} \cdot \mathrm{L}^{-1}$ sodium dodecyl sulfate (SDS) (Acros). Water $\left(18.2 \mathrm{M} \Omega \cdot \mathrm{cm}^{-1}\right)$ was purified and deionized in house via a Milli-Q plus instrument from Millipore (Bedford, New Hampshire, USA). $\mathrm{pH}$ was adjusted to $\mathrm{pH} 7.4$ by phosphate buffer, prepared with $0.05 \mathrm{~mol} \cdot \mathrm{L}^{-1}$ disodium hydrogen phosphate (Sigma-Aldrich) and potassium dihydrogen phosphate (Sigma-Aldrich). To reproduce the osmotic pressure of biological fluids, $\mathrm{NaCl}\left(9.20 \mathrm{~g} \cdot \mathrm{L}^{-1}\right)$ (Sigma-Aldrich) was added to the micellar mobile phase. IAM mobile phases consisted of a solution 70/30 v/v Dulbecco's phosphate-buffered saline (DPBS) / methanol (HPLC-grade; Biosolve, Valkenswaard, The Netherlands). DPBS was composed of $2.7 \mathrm{mmol} \cdot \mathrm{L}^{-1}$ $\mathrm{KCl}, 1.5 \mathrm{mmol} \cdot \mathrm{L}^{-1}$ potassium dihydrogen phosphate, $137.0 \mathrm{mmol} \cdot \mathrm{L}^{-1} \mathrm{NaCl}$, and $8.1 \mathrm{mmol} \cdot \mathrm{L}^{-1}$ disodium hydrogen phosphate (Sigma-Aldrich). Such solution had a pH value of $7.40 \pm 0.05$, and no $\mathrm{pH}$ adjustment was performed. All mobile phases were vacuum-filtered through $0.20 \mu \mathrm{m}$ nylon membranes (Grace, Lokeren, Belgium) before use. Different mobile phases and elution programs were tested starting from 100\% aqueous phase; however, in IAM-LC the latter condition did not allow the elution of the most lipophilic bases in a reasonable amount of time. Stock solutions of all 
453 drugs were prepared by dissolving $10 \mathrm{mg}$ in $1 \mathrm{~mL}$ of methanol except for i) quinidine and 454 theobromine, for which stock concentrations of $1 \mathrm{mg} \cdot \mathrm{mL}^{-1}$ and $200 \mu \mathrm{g} \cdot \mathrm{mL}^{-1}$, respectively, were used, ii) caffeine and theophylline, which were dissolved in water $\left(10 \mathrm{mg} \cdot \mathrm{mL}^{-1}\right)$, iii) domperidone, which was dissolved in dimethyl sulfoxide $\left(10 \mathrm{mg} \cdot \mathrm{mL}^{-1}\right)$ and iv) chlorpromazine, which was dissolved in acetonitrile. Stock solutions were stored at $4{ }^{\circ} \mathrm{C}$, except for atenolol, zidovudine, chlorambucil and rifampicin, which were stored at $-20{ }^{\circ} \mathrm{C}$. Working solutions were freshly 459 prepared at the beginning of each day by dilution, with the mobile phase, of the stock solutions to $46050 \mu \mathrm{g} \cdot \mathrm{mL}^{-1}$ for all the analytes, except for valproic acid and halothane that were diluted to 250 $461 \mu \mathrm{g} \cdot \mathrm{mL}^{-1}$.

\subsection{DATA SOURCES}

Log BB values were taken from the literature ${ }^{18-24} \cdot \mathrm{pKa}$ values were obtained from the literature ${ }^{21}$ except for amobarbital, donepezil, fluphenazine, hydroxyzine, ketorolac, paroxetine and ropinirole, whose values were calculated by the software Marvin Sketch 15.1 for Mac OS X ${ }^{28}$.

\subsection{SOFTWARE}

\subsubsection{MOLECULAR MODELING}

469 Molecular modeling was performed by the software Vega ZZ 3.0.5 for Windows-based PCs ${ }^{31}$. The starting three-dimensional structures of the considered molecules were downloaded from PubChem

471 database ${ }^{32}$ and they were considered in both zero atomic charge and ionized form. The Gasteiger 472 Marsili $^{33}$ method, along with $\mathrm{CHARMM}^{34,35,36}$ force field, was applied to calculate the atomic 473 charges required to perform the next molecular mechanics calculations. An extensive 474 conformational analysis was carried out in vacuum by using the Boltzmann Jump method 475 (MonteCarlo procedure) implemented in AMMP software ${ }^{37}$ which generates 1000 geometries for each compound by randomly rotating the rotors and the obtained lowest energy conformation was

477 further optimized by performing a PM7 semi-empirical calculation with MOPAC 2012 program $^{38}$ 
(keywords: PM7 PRECISE MMOK). A cluster analysis was performed to select the most populated conformation states. Physico-chemical and topological/geometrical properties (Virtual $\log \mathrm{P}^{39}$, lipole $^{40}$, volume, polar surface area, surface accessible to the solvent, gyration radius, ovality, mass, number of atoms, angles, dihedrals, etc) were calculated by VEGA ZZ software and, finally, all molecules were inserted into a Microsoft Access database.

The QSPR models were obtained by the automatic stepwise approach implemented in "Automatic linear regression" script of VEGA ZZ software, calculating regression models, including from 1 to 5 independent variables. The predictive strength of the best equation was evaluated by leave-oneout (LOO) cross validation and the regression coefficients were calculated to evaluate the set in terms of standard deviation of errors, angular coefficient, intercept and $r^{2}$ of the trend line of the chart of the predicted $v s$. experimental activities. Descriptors with too low regression coefficient $\left(r^{2}\right.$ $<0.1)$ were excluded and collinear descriptors were detected by evaluating the variance inflation factor (VIF) whose threshold value was set to 5. A further validation of the model having the highest predictive strength was performed via model validator script, included in Vega.

\subsubsection{MOLECULAR DOCKING}

Molecular docking calculations were carried out using AutoDock 4.2 software ${ }^{41}$. The $3.4 \AA$ resolution P-glycoprotein (P-gp) crystallographic structure (mouse P-glycoprotein 3, gene: MD1A, PDB code: 4Q9H) was downloaded from Protein Data Bank (PDB) Database. Gasteiger partial charges were calculated on ligand atoms. Polar hydrogens were added to P-gp and Gesteiger ${ }^{33}$ partial charges were calculated using AutoDock Tools ${ }^{42}$. Simulation boxes were centered on the ligands in the structures of P-gp-ligand complexes (PDB codes: 4Q9I, 4Q9J, 4Q9K, 4Q9L) as reported in the literature ${ }^{27}$. The simulation boxes were adjusted to accommodate the ligand in each complex and the sizes were between $26 \times 26 \times 26 \AA$ and 30x26x30 $\AA .100$ runs for each simulation were performed and the Lamarckian Genetic Algorithm (number of energy evaluation: $2.5 \times 10^{6}$ ) for the docking simulations was used. The choice was based on the aspect that this protocol 
provides the most efficient search for general applications, and is typically effective for systems with about 10 rotatable bonds in the ligand. The acidic compounds having $\mathrm{pKa}<7.4$ and the basic ones having $\mathrm{pKa}>7.4$ were considered in their dissociated forms. Gasteiger-Marsili ${ }^{33}$ electric 507 charges were supplied. Amphoteric drugs were assumed in their prevalent forms as calculated by 508 the software MarvinSketch ${ }^{28}$. For the analytes supporting one or more stereocenters, the following 509 arrangements were undertaken. When the drugs were administered as racemic mixture (Atenolol, 510 Citalopram, Donepezil, Eserine, Halothane, Hexobarbital, Hydroxyzine, Ibuprofen, Ketorolac, 511 Mianserin, Nicotine, Omeprazole, Oxazepam, Pindolol, Promethazine, Venlafaxine and 512 Verapamil), each stereoisomer was docked into each site of the P-gp and the binding energies 513 presented are the averages of those of the stereoisomers included in the mixtures. On the contrary, 514 when the $\log \mathrm{BB}$ values referred to a specific stereoisomer (Rifampicine, Zidovudine, 515 Levofloxacin) as that was the one administered in the $\log$ BB determinations, only this was docked. 516 When a new stereocenter was created because of protonation, as for instance occurs for tertiary 517 amines supporting different groups, both configurations were tested. The consistency of the results 518 was analyzed by clustering spatially the docked conformations. This step was necessary because of 519 the stochastic nature of the search methods, that are used to predict optimal docked conformations.

\subsection{PROCESSING}

521 The chromatographic retention coefficients of each analytes were calculated by using the following 522 expression:

$$
k=\frac{t_{r}-t_{0}}{t_{0}}
$$

524 in which $t_{\mathrm{r}}$ is the retention time of the compound of interest and $t_{0}$ the retention time of a non525 retained compound (acetone). All reported $\log \mathrm{k}$ values are the average of at least three 526 measurements; for each $\log \mathrm{k}$ value the 95\% confidence interval associated with each value never 
528 Three different sets of properties were generated. At first, all the analytes were considered as 529 uncharged (having full charge equal to 0), subsequently analytes having acidic or basic functions 530 were considered fully ionized and zwitterions were considered with both the acidic and basic 531 functions in their charged forms. Eventually, a weighted average of the static properties at $\mathrm{pH} 7.4$ 532 according to the $\mathrm{pKa}$ of each analyte was performed; for zwitterions, the relative abundance of each 533 microspecies (zero atomic charges, zwitterion, anion and cation) in solution at the physiological $\mathrm{pH}$ 534 (7.4) was calculated by the software Marvin Sketch 15.1 for Mac OS $\mathrm{X}^{28}$. This approach was also 535 extended to the conformational analysis performed in vacuum, yielding three different sets of 536 conformational properties, i.e. i) conformational properties of the forms of the analytes having zero 537 atomic charges, ii) conformational properties of the ionized forms of the analytes, and iii) average 538 of the conformational properties at $\mathrm{pH} 7.4$ according to the $\mathrm{pKa}$ of each analyte and the calculated 539 microspecies distribution for zwitterions. For each of the properties taken into account (Molecular 540 lipophilicity potential (MLP) ${ }^{39}$, lipole ${ }^{40}$, volume, polar surface area, superficial area, gyration 541 radius, ovality, volume diameter, dipolar moment, etc), minimum and maximum value, average, 542 range and standard deviation for each population of conformers were calculated and incorporated in 543 the statistical models. An additional deal of molecular descriptor were calculated by the software E544 Dragon $^{30}$.

\section{Conflict of interest disclosure}

546 The authors declare no competing financial interest.

\section{LIST OF ABBREVIATIONS}

CNS Central Nervous System; D/Dr05 distance/detour ring index of order 5; Impropers Number of improper angles (out of plane); HbDon Number of H-bond donor groups; HbAcc Number of H- 
552 bond acceptor groups; IAM Immobilized artificial membrane; $M D$ Dipole Moment (Debye); $M L C$

553 Micellar liquid chromatography; MLP Molecular Lipophilicity Potential; $n R C O O H$ number of 554 carboxylic group (aliphatic); PLS Partial Least Squares; Psa Polar Surface Area $\left(\AA^{2}\right)$; P-gp P555 glycoprotein; SDS sodium dodecyl sulphate; TPSA (NO) topological polar surface area using N,O 556 polar contributions; TPSA (Tot) topological polar surface area using N,O,S,P polar contributions. 
80

81

\section{Supporting Information}

- $\quad$ Figure 1 Page S-2;

- $\quad$ Figure 2 Page S-4;

- $\quad$ Figure 3 Page S-6;

- raw data in the spreadsheet file "data.xlsx".

- $\quad$ AutoDock GPF file for site 1

- $\quad$ AutoDock DPF file for site 1

- $\quad$ AutoDock GPF file for site 7

- $\quad$ AutoDock DPF file for site 7

To whom correspondence should be addressed:

Prof. Dr. Frederic Lynen

Separation Science Group

Department of Organic and Macromolecular Chemistry

Ghent University

Krijgslaan 281, S4-bis

B-9000 Gent, Belgium

Tel. +(32)9 2649602

Fax +(32)9 2644998

e-mail: frederic.lynen@ugent.be 


\subsection{REFERENCES}

(1) Waring, M.J.; Arrowsmith, J.; Leach, A.R.; Leeson, P.D.; Mandrell, S.; Owen, R.M.; Pairaudeau, G.; Pennie, W.D.; Pickett, S.D.; Wang, J.; Wallace, O.; Weir, A. An Analysis of the Attrition of Drug Candidates from Four Major Pharmaceutical Companies. Nat. Rev. Drug Discov. 2015, 14 (7), 475-486.

(2) Kola, I.; Landis, J. Can the Pharmaceutical Industry Reduce Attrition Rates? Nat. Rev. Drug Discov. 2004, 3 (August), 1-5.

(3) Van Bree, J. B.; De Boer, A. G.; Danhof, M.; Breimer, D. D. Drug Transport across the Blood--Brain Barrier. I. Anatomical and Physiological Aspects. Pharm. Weekbl. Sci. Ed. 1992, $14(5), 305-310$.

(4) Keaney, J.; Campbell, M. The Dynamic Blood-Brain Barrier. FEBS J. 2015, 282 (21), 40674079.

(5) Bickel, U. How to Measure Drug Transport across the Blood-Brain Barrier. NeuroRx 2005, 2 (1), 15-26.

(6) Lundquist, S.; Renftel, M.; Brillault, J.; Fenart, L.; Cecchelli, R.; Dehouck, M.-P. Prediction of Drug Transport through the Blood-Brain Barrier in Vivo: A Comparison between Two in Vitro Cell Models. Pharm. Res. 2002, 19 (7), 976-981.

(7) Ekins, S.; Mestres, J.; Testa, B. In Silico Pharmacology for Drug Discovery: Applications to Targets and Beyond. Br. J. Pharmacol. 2007, 152 (December 2006), 21-37.

(8) Grumetto, L.; Russo, G.; Barbato, F. Indexes of Polar Interactions between Ionizable Drugs and Membrane Phospholipids Measured by IAM-HPLC: Their Relationships with Data of Blood-Brain Barrier Passage. Eur. J. Pharm. Sci. 2014, 65, 139-146.

(9) De Vrieze, M.; Verzele, D.; Szucs, R.; Sandra, P.; Lynen, F. Evaluation of Sphingomyelin, Cholester, and Phosphatidylcholine-Based Immobilized Artificial Membrane Liquid Chromatography to Predict Drug Penetration across the Blood-Brain Barrier. Anal. Bioanal. 
Chem. 2014, 406 (25), 6179-6188.

609 (10) Barbato, F.; Di Martino, G.; Grumetto, L.; La Rotonda, M. I. Prediction of Drug-Membrane Interactions by IAM-HPLC: Effects of Different Phospholipid Stationary Phases on the Partition of Bases. Eur. J. Pharm. Sci. 2004, 22 (4), 261-269.

(15) Verzele, D.; Lynen, F.; Vrieze, M. D.; Wright, A. G.; Hanna-Brown, M.; Sandra, P. Development of the First Sphingomyelin Biomimetic Stationary Phase for Immobilized Artificial Membrane (IAM) Chromatography. Chem. Commun. 2012, 48 (8), 1162-1164.

(16) De Vrieze, M.; Lynen, F.; Chen, K.; Szucs, R.; Sandra, P. Predicting Drug Penetration across the Blood-Brain Barrier: Comparison of Micellar Liquid Chromatography and Immobilized Artificial Membrane Liquid Chromatography. Anal. Bioanal. Chem. 2013, 405, 6029-6041.

(17) Berthod, A.; Garcia-Alvarez-Coque, C. Micellar Liquid Chromatography; Marcel Dekker Incorporated, New York, 2000. 
(18) Abraham, M. H.; Chadha, H. S.; Whiting, G. S.; Mitchell, R. C. Hydrogen Bonding. 32. An Analysis of Water-Octanol and Water-Alkane Partitioning and the Deltalog P Parameter of Seiler. J. Pharm. Sci. 1994, 83 (8), 1085-1100.

(19) Katritzky, A. R.; Kuanar, M.; Slavov, S.; Dobchev, D. A.; Fara, D. C.; Karelson, M.; Acree, W. E.; Solov'ev, V. P.; Varnek, A. Correlation of Blood-Brain Penetration Using Structural Descriptors. Bioorganic Med. Chem. 2006, 14 (14), 4888-4917.

(20) Platts, J. A.; Abraham, M. H.; Zhao, Y. H.; Hersey, A.; Ijaz, L.; Butina, D. Correlation and Prediction of a Large Blood-Brain Distribution Data Set - An LFER Study. Eur. J. Med. Chem. 2001, 36 (9), 719-730.

(21) Avdeef, A. Absorption and Drug Development; John Wiley \& Sons, Inc.: Hoboken, NJ, USA, 2012; pp 625-663.

(22) Mente, S. R.; Lombardo, F. A Recursive-Partitioning Model for Blood-Brain Barrier Permeation. J. Comput. Aided. Mol. Des. 2005, 19 (7), 465-481.

(23) Abraham, M. H.; Ibrahim, A.; Zhao, Y.; Jr, W. E. A.; Al, A. E. T. A Data Base for Partition of Volatile Organic Compounds and Drugs From Blood / Plasma / Serum to Brain, and an LFER Analysis of the Data. J. Pharm. Sci. 2006, 95 (10), 2091-2100.

(24) Björkman, S. Prediction of the Volume of Distribution of a Drug: Which Tissue-Plasma Partition Coefficients Are Needed? J. Pharm. Pharmacol. 2002, 54 (9), 1237-1245.

(25) Avdeef, A. Absorption and Drug Development; John Wiley \& Sons, Inc.: Hoboken, NJ, USA, 2012; p 595-602 and references cited therein.

(26) Eyal, S.; Hsiao, P.; Unadkat, J. D. Drug Interactions at the Blood-Brain Barrier: Fact or Fantasy? Pharmacol. Ther. 2009, 123 (1), 80-104.

(27) Szewczyk, P.; Tao, H.; McGrath, A. P.; Villaluz, M.; Rees, S. D.; Lee, S. C.; Doshi, R.; Urbatsch, I. L.; Zhang, Q.; Chang, G. Snapshots of Ligand Entry, Malleable Binding and Induced Helical Movement in P-Glycoprotein. Acta Crystallogr. Sect. D Biol. Crystallogr. 2015, 71, 732-741. 
(28) ChemAxon. Marvin Sketch.

(29) Vistoli, G.; Pedretti, A.; Testa, B. Partition Coefficient and Molecular Flexibility: The Concept of Lipophilicity Space. Chem. Biodivers. 2009, 6 (8), 1152-1169.

(30) Tetko, I. V; Gasteiger, J.; Todeschini, R.; Mauri, A.; Livingstone, D.; Ertl, P.; Palyulin, V. A.; Radchenko, E. V; Zefirov, N. S.; Makarenko, A. S.; Tanchuk, V. Y.; Prokopenko, V. V. Virtual Computational Chemistry Laboratory--Design and Description. J. Comput. Aided. Mol. Des. 2005, 19 (6), 453-463.

(31) Pedretti, A.; Villa, L.; Vistoli, G. VEGA - An Open Platform to Develop Chemo-BioInformatics Applications, Using Plug-in Architecture and Script Programming. J. Comput. Aided. Mol. Des. 2004, 18 (3), 167-173.

(32) Kim, S.; Thiessen, P. A.; Bolton, E. E.; Chen, J.; Fu, G.; Gindulyte, A.; Han, L.; He, J.; He, S.; Shoemaker, B. A.; Wang, J.; Yu, B.; Zhang, J.; Bryant, S. H. PubChem Substance and Compound Databases. Nucleic Acids Res. 2016, 44 (D1), D1202-1213.

(33) Gasteiger, J.; Marsili, M. Iterative Partial Equalization of Orbital Electronegativity-a Rapid Access to Atomic Charges. Tetrahedron 1980, 36 (22), 3219-3228.

(34) Brooks, B. R.; Brooks, C. L., 3rd; Mackerell, A. D., Jr.; Nilsson, L.; Petrella, R. J.; Roux, B.; Won, Y.; Archontis, G.; Bartels, C.; Boresch, S.; Caflisch, A.; Caves, L.; Cui, Q.; Dinner, A. R.; Feig, M.; Fischer, S.; Gao, J.; Hodoscek, M.; Im, W.; Kuczera, K.; Lazaridis, T.; Ma, J.; Ovchinnikov, V.; Paci, E.; Pastor, R. W.; Post, C. B.; Pu, J. Z.; Schaefer, M.; Tidor, B.; Venable, R. M.; Woodcock, H. L.; Wu, X.; Yang, W.; York, D. M.; Karplus, M. CHARMM: the biomolecular simulation program. J. Comput. Chem. 2009, 30, 1545-1614.

(35) Brooks, B. R.; Bruccoleri, R. E.; Olafson, B. D.; States, D. J.; Swaminathan, S.; Karplus, M. CHARMM: A program for macromolecular energy, minimization, and dynamics calculations. J. Comput. Chem. 1983, 4, 187-217.

(36) MacKerell, A. D.; Brooks, B.; Brooks, C. L.; Nilsson, L.; Roux, B.; Won, Y.; Karplus, M. CHARMM: The Energy Function and Its Parameterization with an Overview of the Program. 
In Encyclopedia of Computational Chemistry, 1st ed.; Schleyer, P. v. R., Ed.; John Wiley \& Sons, Inc.: Chichester, UK, 1998; 271-277.

688

689 (37) Harrison, R. W. Stiffness and Energy-Conservation Molecular-Dynamics - An Improved Integrator. J. Comput. Chem. 1993, 14 (9), 1112-1122.

691

(38) Stewart, J. J. P. MOPAC: A Semiempirical Molecular Orbital Program. J. Comput. Aided. 692 Mol. Des. 1990, 4 (1), 1-103.

693

(39) Gaillard, P.; Carrupt, P. A.; Testa, B.; Boudon, A. Molecular Lipophilicity Potential, a Tool in 3D QSAR: Method and Applications. J. Comput. Aided. Mol. Des. 1994, 8 (2), 83-96. 695

(40) Pedretti, A.; Villa, L.; Vistoli, G. Modeling of Binding Modes and Inhibition Mechanism of 696 697 698 699 700 701 Some Natural Ligands of Farnesyl Transferase Using Molecular Docking. J. Med. Chem. 2002, 45 (7), 1460-1465.

(41) Huey, R.; Morris, G. M.; Olson, A. J.; Goodsell, D. S. Software News and Update. A Semiempirical Free Energy Force Field with Charge-Based Desolvation. J. Comput. Chem. 2007, 28, 1145-1152.

(42) Morris, G. M.; Ruth, H.; Lindstrom, W.; Sanner, M. F.; Belew, R. K.; Goodsell, D. S.; Olson, A. J. Software News and Updates AutoDock4 and AutoDockTools4: Automated Docking with Selective Receptor Flexibility. J. Comput. Chem. 2009, 30 (16), 2785-2791. 
708 TABLE OF CONTENTS GRAPHIC

709

710

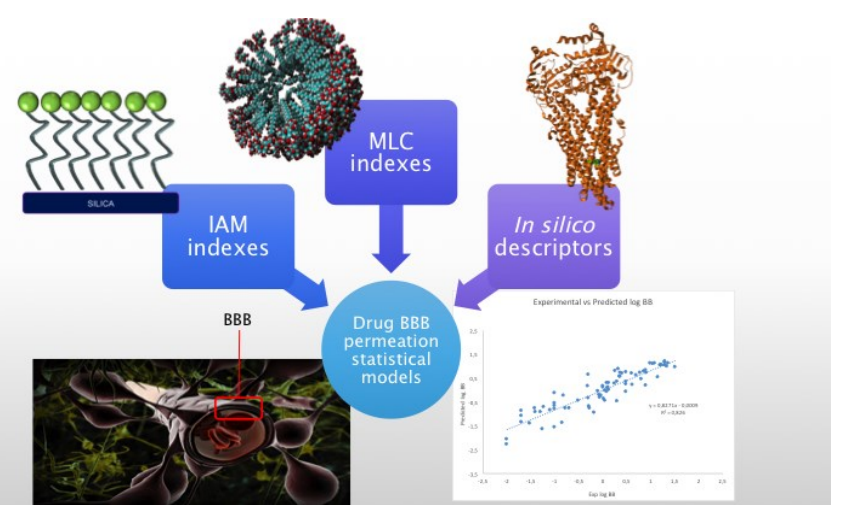

